\title{
A Constellation of Eye-Tracking Measures Reveals Social Attention Differences in ASD and the Broad Autism Phenotype
}

\section{Kritika Nayar}

Northwestern University

Frederick Shic

Seattle Children's Research Institute

\section{Molly Winston}

Northwestern University

Molly Losh ( $\nabla$ m-losh@northwestern.edu )

Northwestern University https://orcid.org/0000-0002-9823-8249

\section{Research}

Keywords: eye tracking, autism spectrum disorder, broad autism phenotype, social attention, visual processing, endophenotype.

Posted Date: September 14th, 2021

DOl: https://doi.org/10.21203/rs.3.rs-859251/v1

License: (c) (1) This work is licensed under a Creative Commons Attribution 4.0 International License. Read Full License 


\section{Abstract}

Background: Social attention differences, expressed through gaze patterns, have been documented in autism spectrum disorder (ASD), with subtle differences also reported among first-degree relatives, suggesting a shared genetic link. Findings have mostly been derived from standard eye-tracking methods (total fixation count or total fixation duration). Given the dynamics of visual attention, these standard methods may obscure subtle, yet core, differences in visual attention mechanisms, particularly those presenting sub-clinically. This study applied a constellation of eye-tracking analyses to gaze data from individuals with ASD and their parents.

Methods: This study included $n=156$ participants across groups, including ASD $(n=24)$ and control $(n=32)$ groups, and parents of individuals with ASD $(n=61)$ and control parents $(n=39)$. A complex scene with social/non-social elements was displayed and gaze tracked via an eye tracker. Eleven analytic methods from the following categories were analyzed: 1) standard variables, 2) temporal dynamics (e.g., gaze over time), 3) fixation patterns (e.g., perseverative or regressive fixations), 4) first fixations, and 5) distribution patterns. MANOVAs, growth curve analyses, and Chi-squared tests were applied to examine group differences. Finally, group differences were examined on factor scores derived from a principal component analysis (PCA) that reduced variables to distinct dimensions.

Results: No group differences emerged among standard, first fixation, and distribution pattern variables. Both the ASD and ASD parent groups demonstrated reduced social attention over time and atypical perseverative fixations. Lower social attention factor scores derived from PCA strongly differentiated the ASD and ASD parent groups from controls, with parent findings driven by the subset of parents demonstrating the broad autism phenotype.

Limitations: To generalize these findings, larger sample sizes, extended viewing contexts (e.g., dynamic stimuli), and even more eye-tracking analytical methods are needed.

Conclusions: Fixations over time and perseverative fixations differentiated ASD and the ASD parent groups from controls, with the PCA most robustly capturing social attention differences. Findings highlight their methodological utility in studies of the (broad) autism spectrum to capture nuanced visual attention differences that may relate to clinical symptoms in ASD, and reflect genetic liability in clinically unaffected relatives. This proof-of-concept study may inform future studies using eye tracking across populations where social attention is impacted.

\section{Introduction}

The study of eye movement patterns has proven to be a powerful tool for revealing meaningful information about perceptual and attentional strategies, including providing an indirect measurement of underlying cognitive, attentional, and executive skills (1). Early eye-tracking studies (2), along with many 
that followed (1,3-6), have demonstrated that the location of gaze (i.e., where individuals look when visually exploring stimuli) not only reflects attentional processes $(7,8)$, but also maps onto underlying thoughts and cognition (1). In this way, eye movement may be considered as both complementary to and associated with both psychophysical (e.g., accuracy and reaction time indices) and neural measurements of cognition (9). For example, studies have demonstrated that saccade speed or saccade target location predicts reaction time $(10,11)$, and that analyses of gaze could index activity in areas of the brain associated with visual perception (e.g., visual examination of social stimuli activates the amygdala, and face perception activates the Fusiform Face Area). (12-16). Eye-tracking also has the potential to reveal moment-to-moment information of underlying cognition, revealing nuanced and dynamic patterns at an individual or group level. Given the often automatic and rapid nature of eye movements, analysis of gaze may thus represent a phenomenon existing as an intermediate link between brain and behavior, with the potential of revealing cognitive differences that may stem from underlying neurobiology.

The use of eye tracking has been particularly revealing in studies of social cognition in autism spectrum disorder (ASD) (17-19). Specifically, differences in visual attention have been repeatedly documented in individuals ASD relative to controls (20-22), with differences relating to social communication impairments $(17,23-26)$ and restricted and repetitive behaviors $(27,28)$, core features of the disorder. Gaze and eye movement differences can be detected as early as infancy in individuals with ASD (such as lacking a preference between looking at faces versus objects, or showing atypical scanning patterns when gazing at faces) (29-36). These visual attention differences appear to persist into adolescence and adulthood, wherein individuals with ASD have been reported to show slower latencies to orient to social features of the scene $(28,37)$ and exhibit different scanning patterns $(21,38)$; though findings vary depending on the type of stimulus $(26,39)$. For example, when there is competing information in the scene (i.e., both salient and non-salient features), individuals with ASD tend to spend more time exploring non-salient aspects of the scene, such as inanimate objects or non-eye regions of facial stimuli $(21,23$, $40,41)$. These differences appear to be clinically meaningful, with several studies reporting a link between atypical gaze patterns and reduced social competency $(17,30)$.

Eye movement patterns appear to be heritable in the general population (42) and across psychiatric disorders (43), suggesting that eye movements and visual attention may not only reflect underlying genetics (i.e., constituting endophenotypes, which are heritable characteristics associated with the genetic underpinnings of a disorder (44)), but also neurobiological mechanisms contributing to the development of a disorder's symptomatology. Studies of gaze in individuals with ASD and their relatives thus have the potential to inform the mechanistic processes related to the etiology and development of ASD symptomatology, as well as subclinical features of the broad autism phenotype (BAP), which are linked to underlying genetic liability in clinically unaffected relatives (45-47). Indeed, subtle differences in gaze have been reported among first-degree relatives of individuals with $\operatorname{ASD}(23,42,47,48)$, and particularly among those who display the BAP (48-51). For instance, Adolphs et al., (48), showed that parents who displayed features of the BAP relied less on the eye region of the face when making judgments about emotions. Less fluent gaze-language coordination and subtle differences in gaze when narrating from illustrated stimuli have also been reported among parents, and linked with BAP features $(23,27)$. 
Together, this work demonstrates the utility of studying looking patterns as potentially good candidate endophenotypes, given their known heritability and association with ASD. However, visual attention and related eye movement patterns have typically been examined using primarily global fixation measures across many different stimuli across studies, and have been understudied in first-degree relatives with only three studies to date examining visual perception/attention using eye tracking in parents $(23,27,51)$. Further study is therefore warranted to understand the nature and extent of gaze differences in relatives, how such differences may overlap with those evident in ASD, and important connections to underlying cognitive mechanistic and biological factors. Additionally, evidence suggests that study results dependent on standard fixation measures are heavily influenced by processing methods (52-54), and have been shown to dramatically alter findings in ASD (54). Finally, these standard measures provide only a global overview of looking patterns, and may miss potentially important subtleties evident in ASD and the BAP.

Ongoing studies have demonstrated the efficacy of applying other methods of analyses to examine eyetracking data, which may reveal important aspects of cognition. For example, perseverations (repeat successive fixations towards the same area of interest) may reflect "sticky attention" or mental disengagement (55), whereas regressions (i.e., refixating on previously examined areas of interest) may index the loss of mental set or executive control, as the looker's attention is captured (56-59). Analysis of both these fixation types have been fruitfully applied in studies of ASD and the BAP in studies using social and non-social stimuli, and were also found to relate to ASD symptomatology $(27,28)$. Shic and colleagues (41) utilized another fixation analytic method, transition entropy analyses, in young children with ASD, finding no changes in the transitions of fixations (i.e., transitions from one AOI to another) between salient and non-salient regions of the face in individuals with ASD, unlike controls, thus demonstrating differences in patterns of attention allocation between groups. Spatial Distribution Analysis or Nearest Neighbor Index (i.e., distance-dispersion algorithm) were also examined in children with and without ASD, to explore how fixations were dispersed across the facial stimuli (60). Finally, growth curve analyses (GCA), proves to be a rigorous method of assessing changes over the course of time, which can be applied to understand the moment-to-moment pattern of gaze while interpreting a scene. In particular, GCA maps out the time-linked gaze trajectory over the course of the stimulus presentation and can elucidate changes occurring longitudinally across developmental time periods, such as those found in infants with and without ASD (61). As such, while traditional methods of dwell time and fixations can reveal important aspects of information processing, they may lack sensitivity for capturing more nuanced patterns of eye movement that can be revealed through more detailed and expansive methods.

The present study was an attempt to address this concern, through application of a suite of eye tracking analytic tools to gaze data in ASD and the BAP, with the goal of evaluating potentially more sensitive methods of gaze patterns than global analyses of looking time or fixations, and that might help to capture subtle top-down social visual attentional differences in non-clinical populations such as the BAP. Based on evidence previously reviewed and the social atypicalities inherent to ASD, and more subtly in the BAP, it was predicted that individuals with ASD and their parents (particularly those with features of the 
BAP) would show reduced social attention and increased attention to non-salient components of the scene, reflective of decreased attribution of social salience. Based on evidence demonstrating relationships between perseverative eye movement patterns and restricted and repetitive behaviors, a larger number of refixations (repeated fixations) and shorter first fixation duration was also expected, with decreased spatial distribution of fixations, and atypical fixation transition and looking patterns over the course of the task compared to controls.

\section{Methods}

\section{Participants (Table 1)}


Table 1

Sample Characteristics

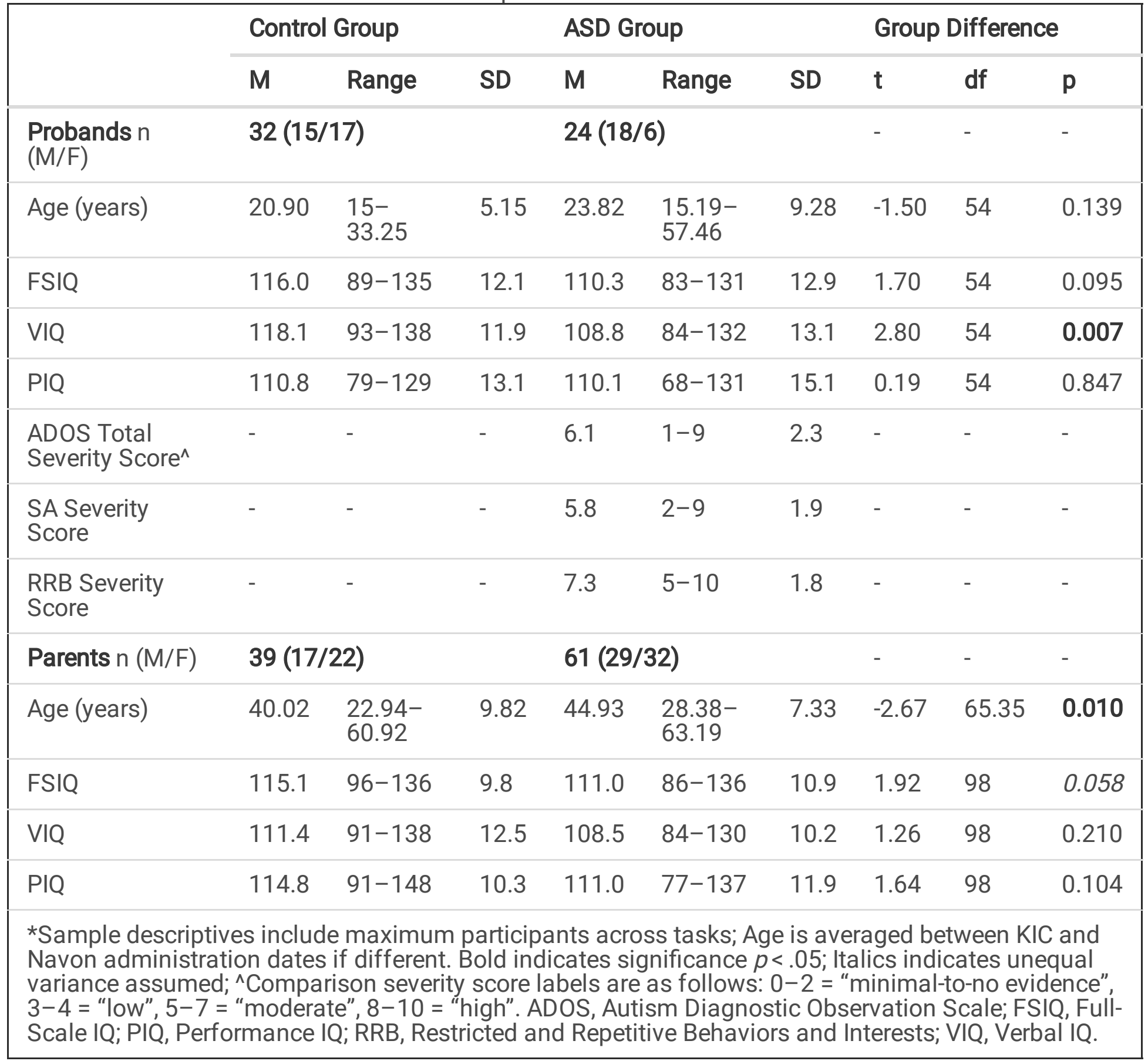

Participants included in the present study were identical to those included in a prior study exploring language and related looking patterns (23). Twenty-nine individuals with ASD (ASD group) and 34 control participants (control group), as well as 74 parents of individuals with ASD (ASD parent group) and 45 control parents (control parent group) were included in the study. For details pertaining to screening inclusionary and exclusionary criteria, see Lee et al., 2019. Participants were excluded for any severe psychiatric disorder (e.g., schizophrenia, bipolar disorder) and uncorrected vision impairments (e.g., strabismus). Participant characteristics are outlined in Table 1. All procedures were approved by the University's Institutional Review Board and written informed consent/assent were obtained for all participants. 
Individuals with ASD were included following confirmation of ASD with gold standard instruments (Autism Diagnostic Observation Schedule-General or 2nd Edition (ADOS) (62) and/or the Autism Diagnostic Interview-Revised (ADI-R) (63)). Parents of an individual with ASD were included if they had at least one child with idiopathic ASD, and control participants were required to have no personal or family history of ASD or related genetic disorders (e.g., fragile X syndrome). BAP status was assessed in the ASD parent group only, given low base rates of the BAP among individuals without a family history of ASD (49, 64,65). BAP status was assessed using the Modified Personality Assessment Scale-Revised (MPAS-R) (64), which includes a series of questions specifically designed to tap the subclinical features related to the BAP including aloof, rigid, perfectionistic, and untactful personality traits. Coding of personality features followed methods outlined in prior work, such that raters were assigned scores ranging from 0 to 2 (trait absent, possibly present, definitely present) on a 5-point Likert scale.

\section{Eye tracking procedures}

\section{General procedures}

Participants were asked to narrate a story after looking at an image being presented for 8 seconds on a 17-inch TFT LCD (1,280 x 1,024 resolution) Tobii T60 series eye tracker (Tobii Technology AB, Danderyd, Sweden). All participants were seated $50-60 \mathrm{~cm}$ from the screen and had their gaze calibrated prior to task administration, including using a standard 5-point calibration grid, which has a visual angle accuracy of $0.5^{\circ}$. Participants were recalibrated following any large movements. Tracking was monitored live during task administration using Tobii Studio's built-in live view and track window options, with additional calibration checks embedded in the task (e.g., center crosshair, corner star) to ensure tracking accuracy.

\section{Eye tracking task}

The Thematic Apperception Test (TAT) (66) was developed as a psychological projective test, and has been used in numerous studies of narrative elicitation $(23,67-70)$. Prior work (23) has demonstrated gaze differences in ASD and ASD parent groups when generating stories from TAT stimuli. The current study focused on the "Farmland Scene" (greyscale card 2; Fig. 1A) from the TAT, because of its complexity and prior findings that global indices of fixation revealed differences in attention to setting and protagonists among individuals with ASD and their parents with the BAP (23).

\section{Data Processing}

Areas of Interests (AOIS). AOls were manually drawn in Tobii Studio. The AOIs in the Farmland Scene are depicted in Fig. 1B, and were categorized as either social (e.g., human figures) or non-social (e.g., barn in the background). "Buffer" regions were created identical to prior work, such that each AOI was proportionally expanded by up to $10 \%$ of its original size (71). When social and non-social AOls overlapped, the final AOI designation was assigned as social. 
Gaze processing parameters. Eye movements were recorded for both eyes with a sampling rate of $60 \mathrm{~Hz}$. Parameters to account for data loss in working with populations with neurodevelopmental disorders were modeled in line with previous pipelines (53). These parameters were consistent with prior work (23) and the built-in I-VT fixation filter in Tobii Studio as follows-1) fixations were based on the strict average across both eyes; 2 ) a velocity threshold of 35 degrees per second was established; 3 ) adjacent fixations were merged if fixations were less than $100 \mathrm{~ms}$ apart and angles were less than 0.5 degrees apart; 4) missing data were linearly interpolated based on a 150 ms maximum continuous gap; and 5) noise reduction was addressed by utilizing a moving average window of 3 samples. Finally, following data export, fixations were set to be a minimum duration of $100 \mathrm{~ms}$ (i.e., fixation durations less than $100 \mathrm{~ms}$ were excluded).

Quality control procedures. Track loss was based on prior work such that participants' data were excluded if their overall fixation count on the Farmland Scene was $<5$ and the total fixation duration was $<4$ seconds (i.e., gaze data was reliably collected for at least half of the 8 seconds that the stimulus was presented). These criteria resulted in the exclusion of $17 \%$ ASD $(n=5), 6 \%$ control $(n=2), 18 \%$ ASD-parent $(n=13)$ and $13 \%$ control parent $(n=6)$ participants' data. Fisher's Exact Test (FET) and Chi-Squared Test revealed no significant group differences in the proportion of valid or invalid data in ASD versus controls (FET $p=.233$ ) or in ASD parents versus control parents $\left(X^{2}(1,119)=.374, p=.541\right)$, respectively. The final sample was as follows: $n=29$ in the ASD group, $n=34$ in the control group, $n=61$ in the ASD parent group, and $n=39$ in the control parent group. From the sample of parents of individuals with ASD, 24 were characterized as $\mathrm{BAP}(+)$ (i.e., meeting criteria for having BAP traits), and 37 were characterized as $\mathrm{BAP}(-)$ (i.e., not meeting criteria for the BAP).

\section{Eye-tracking variables}


Table 2

Definitions eye-tracking variables

\section{Variable}

Variable Definition

\section{Standard gaze variables}

(1) Dwell time*

Percentage of looking time (sec) toward an $\mathrm{AOI}$ was derived by summing the fixation duration of each $\mathrm{AOI}$ and dividing it by the total duration of looking, multiplied by 100 .

(2) Fixation count*

Percentage of the number of fixations was captured by summing the total number of fixations toward an $\mathrm{AOI}$ out of the total number of fixations across the duration of stimulus presentation, multiplied by 100 .

\section{Temporal dynamics}

(3) Percentage of fixations over time
Growth curve analyses (GCA) were employed to investigate change in looking patterns (percentage of fixations) over the course of the stimulus towards social versus non-social AOls. To account for track loss at the beginning and end of the stimulus presentation, 7 seconds of the 8 second image were examined ( $500 \mathrm{~ms}$ removed from the beginning and end of the stimuli), using 1 second time bins. Follow-up analyses examined the divergence between groups of social versus non-social looking using t-tests with 300 ms time bins.

\section{Fixation patterns}

(4) Perseverative fixations*

(5) Regressive fixations*
Percentage of perseverative fixations were derived by summing fixations that occurred in succession toward the same AOI, divided by the total number of fixations, multiplied by 100 .

Percentage of regressive fixations was captured as the percentage of times a participant returned their gaze to a specific AOI that had already been previously explored, by summing the number of fixations that occurred towards an AOI previously fixated (not including successive fixations/perseverative fixations), divided by the total number of fixations, multiplied by 100 .
(6) Fixation transition analysis*
Transitions between social and non-social information were explored in four ways: i) social to social AOI transitions, ii) non-social to non-social AOI transitions, iii) social to non-social AOI transitions, iv) non-social to social AOI transitions, and v) total transitions between social and non-social AOls. Percentages based on the total number of transitions information were calculated for i) - iv).

\section{First fixations}

(7) First fixation AOI

The percentage of first fixations toward social or non-social information was measured by summing the total number of first fixations that was social or non-social and dividing it by the total number of first fixations, multiplied by 100 .

(8) First fixation duration*
The first fixation duration was derived by measuring the time (in sec) spent examining any $\mathrm{AOI}$ during the first fixation (i.e., the first fixation that occurs after the stimulus appears) before making a fixation transition.

\section{Distribution patterns}




\begin{tabular}{|ll|}
\hline Variable & Variable Definition \\
\hline $\begin{array}{l}\text { (9) Fixation rate } \\
\text { exploration)* }\end{array}$ & $\begin{array}{l}\text { The total number of fixations per participant was divided by the total time } \\
\text { spent examining the scene, to produce the number of fixations that occurred } \\
\text { per second of track time regardless of AOI. }\end{array}$ \\
\hline $\begin{array}{l}\text { (10) Fixation rate } \\
\text { exploration) AOI* }\end{array}$ & $\begin{array}{l}\text { The number of fixations per track time (in sec) toward social or non-social } \\
\text { information was calculated. }\end{array}$ \\
\hline $\begin{array}{l}\text { (11) Fixation spatial } \\
\text { distribution/coverage* }\end{array}$ & $\begin{array}{l}\text { First, a } 5 \times 4 \text { matrix of } 20 \text { large areas }\left(256 \times 256 \text { pixels } / 6.45^{\circ} \times 6.45^{\circ}\right) \\
\text { a } 10 \times 8 \text { matrix of } 80 \text { small areas }\left(128 \times 128 \text { pixels } / 3.2^{\circ} \times 3.2^{\circ}\right) \text { were } \\
\text { generated. Each fixation point's location was categorized into one of these }\end{array}$ \\
$\begin{array}{l}\text { 20 or } 80 \text { "boxes", respectively. To account for the different number of } \\
\text { fixations per participant, the percentage of mini areas explored was } \\
\text { computed per participant by taking the number of areas explored and } \\
\text { dividing it by the total number of fixations for that participant, which was } \\
\text { then multiplied by } 100 \text {. This final percent coverage was included in } \\
\text { subsequent analyses for larger }(5 \times 4 \text { matrix) and smaller }(10 \times 8 \text { matrix }) \\
\text { areas. }\end{array}$ \\
\hline * Variables included in the principal component analysis \\
\hline
\end{tabular}

\section{Standard gaze variables}

Dwell time and fixation count were included in an existing study (Lee et al., 2019), and re-reported here for the purpose of examining the efficacy of more nuanced eye-tracking variables in relation to the overall gaze variables. The below metrics are thought to reflect attentional engagement as well as processing time (1).

(1) Dwell time-Percentage of looking time (sec) toward an AOI was derived by summing the fixation duration of each AOI and dividing it by the total duration of looking, multiplied by 100 .

(2) Fixation count-Percentage of the number of fixations was captured by summing the total number of fixations toward an $\mathrm{AOI}$ out of the total number of fixations across the duration of stimulus presentation, multiplied by 100 .

\section{Temporal Dynamics:}

(3) Fixations over time-Growth curve analyses (GCA) were employed to investigate change in looking patterns (percentage of fixations) over the course of the stimulus presentation towards social versus nonsocial AOls, adapted from the EyetrackingR package (72). To account for track loss at the end of the image as well as pre-established attention-capturing stimuli (i.e., center and corner crosshairs) prior to stimulus presentation, 7 seconds of the 8 second image were examined (500 ms removed from the beginning and end of the stimuli), using 1 second time bins. Divergence analyses were then applied to examine at which point(s) groups differed from one another in terms of social versus non-social looking. Divergence analyses applied t-tests that are embedded within the divergence vignette from the EyetrackingR package (72). Time bins were set to $300 \mathrm{~ms}$ to match the approximate average fixation duration across participants. Taken together, these sets of analyses not only provide information on the 
dynamic patterns of looking via GCA, but also delineate the exact moments in which groups diverge from one another.

\section{Fixation patterns:}

(4) Perseverative fixations-Percentage of perseverative fixations were derived by summing fixations that occurred in succession toward the same AOI, divided by the total number of fixations, multiplied by 100 . Perseverative fixations are thought to tap into attentional "stickiness" or mental disengagement (55).

(5) Regressive fixations-Percentage of regressive fixations was captured as the percentage of times a participant returned their gaze to a specific $\mathrm{AOI}$ that had already been previously explored, by summing the number of fixations that occurred towards an AOI previously fixated (not including successive fixations/perseverative fixations), divided by the total number of fixations, multiplied by 100 . Regressions are thought to reflect slower processing speed or the loss of mental set/executive control (56-59), and reflects the information that repeatedly attracted the viewer's attention.

(6) Fixation transition analysis - Examining transitions between different AOls provides an estimate of general exploration. For the purpose of this study, to demonstrate the utility of examining transitions, we explored the transitions between social and non-social information in four ways: i) social to social AOI transitions, ii) non-social to non-social AOI transitions, iii) social to non-social AOI transitions, and iv) nonsocial to social AOI transitions. Percentages based on the total number of transitions were calculated for i) - iv). Fixation transition has been shown to reflect shifts in attention (9), in addition to reasoning abilities through the process of comparison between task relevant and task irrelevant information (7375).

\section{First fixations:}

(7) First fixation AOI-The percentage of first fixations toward social or non-social information was measured by summing the total number of first fixations that was social or non-social and dividing it by the total number of first fixations, multiplied by 100 . First fixation $\mathrm{AOI}$ is thought to index the utilization of peripheral visual information, that is associated with global or rapid and automatic visual information processing and generally reflects visual information preference (76).

(8) First fixation duration-The first fixation duration was derived by measuring the time (in sec) spent examining any $\mathrm{AOI}$ during the first fixation (i.e., the first fixation that occurs after the stimulus appears) before making a fixation transition. The first fixation duration on an AOI reflects how much either social or non-social information initially attracted the looker's attention.

\section{Distribution patterns:}

(9) Fixation rate (exploration) - In order to obtain a general measure of fixation rate (i.e., exploration) regardless of $\mathrm{AOI}$ type was examined. The total number of fixations per participant was divided by the total time spent examining the scene, to produce the number of fixations that occurred per second of 
track time regardless of $\mathrm{AOI}$. A higher number indicates a greater number of fixations occurring per second of track time, reflecting greater exploration or fixation rate.

(10) Fixation rate (exploration) AOI-To investigate how much participants explored either social or nonsocial AOls, the number of fixations per track time (in sec) toward social or non-social information was further explored. Greater exploration reflects a larger number of fixations toward social or non-social information for a given second, and provides a general indication of attentional capacity and cognitive load (77).

(11) Fixation spatial distribution / coverage analyses (Fig. 2)-Spatial distribution/coverage analyses were conducted to obtain an estimate of how much of the screen was being explored regardless of social or non-social AOI. Given that the Tobii T60 screen was 1,280 x 1,024 pixels, a $5 \times 4$ matrix of 20 large areas $\left(256 \times 256\right.$ pixels $\left./ 6.45^{\circ} \times 6.45^{\circ}\right)$ and a $10 \times 8$ matrix of 80 small areas $\left(128 \times 128\right.$ pixels $/ 3.2^{\circ} \mathrm{x}$ $3.2^{\circ}$ ) were generated. Based on prior work (78) showing that the attention maintaining and capturing abilities of an $\mathrm{AOI}$ increases with size but asymptotes at $3^{\circ}$ visual angle (i.e., $120 \times 120$ pixels using the T60 Tobii display) for ASD and control groups, the smaller area $10 \times 8$ matrix would be the most appropriate for the present study while still maintaining equal sized "boxes". Each fixation point's location was categorized into one of these 20 or 80 "boxes", respectively. To account for the different number of fixations per participant, the percentage of mini areas explored was computed per participant by taking the number of areas explored and dividing it by the total number of fixations for that participant, which was then multiplied by 100 . This final percent coverage was included in subsequent analyses for larger ( 5 x 4 matrix) and smaller ( $10 \times 8$ matrix) areas. A higher percentage represents greater coverage overall (i.e., a greater proportion of fixations were covering unique areas not previously explored), while a lower percentage indexes less coverage or scatter. This measure indicates whether visual attention was "trapped" within certain general regions of a stimulus, or whether there is greater flexibility in underlying attentional mechanisms.

\section{Statistical analyses \\ General analytic plan}

All analyses were conducted using the following comparisons: i) ASD group versus control group; ii) ASD parent group versus control parent group; and iii) $\mathrm{BAP}(+)$ versus $\mathrm{BAP}(-)$ versus control parent group, to examine the role of BAP status on overall group differences among parents.

Assumptions testing.

Data were examined to ensure model assumptions of primary statistical tests (i.e., multivariate analysis of variance; MANOVA) were met. Gaze variables for social and non-social looking were included in assumptions testing, and were conducted separately for proband and parent groups. All assumptions were adequately, with the exception of homogeneity of variance-covariance matrices for some variables 
and sphericity assumptions for others. As such, across all variables, findings using the more robust Pillai's Trace (79) are reported.

Group differences, and growth curve analyses.

Group differences in standard gaze variables, fixation patterns, and fixation rates were examined using a one-way MANOVA separately for proband and parent (including by BAP status) groups, with social and non-social AOIs as the dependent variables. Only significant MANOVAs were followed up with univariate ANOVAs. For BAP-level differences, additional planned post-hoc pairwise comparisons (i.e., BAP $(+)$ versus BAP(-) versus control parent groups) were conducted when the overall MANOVA or univariate ANOVAs were significant. Given that the percent of perseverations towards social and non-social information was skewed, MANOVA results were followed up with non-parametric Mann-Whitney $U$ tests. To examine gaze variables not involving social and non-social AOls (i.e., fixation spatial distribution/coverage, first fixation duration), one-way ANOVAs were conducted separately for proband and parent groups.

Due to their lower sample sizes and categorical nature, first fixation AOls were analyzed using chi-squared tests. Specifically, because sample sizes were low for first fixations towards non-social information $(n=6$ individuals with ASD/controls and $n=17$ parents of individuals with ASD/control parents), a series of $2 X$ 2 contingency tables using Fisher's exact tests were performed separately in parent and proband groups to examine group differences in the proportions of first fixations that were directed towards social and non-social AOI.

To investigate changes in looking patterns towards social versus non-social AOls over the course of the stimulus presentation, growth curve analyses (GCA) were utilized using similar methods applied in recent work examining gaze during affective facial expressions (80). Specifically, orthogonal polynomial terms, each representing a different pattern of looking, were added in a stepwise fashion: 1) the linear term reflected an increase or decrease in proportion of looking over time linearly; 2) the quadratic term reflected the dynamic nature of switching from one AOI to another and back again; and 3) the cubic term reflected the timing of switches between AOls. Only interactions that include a polynomial term were reported.

Finally, a principal component analysis (PCA) was applied to determine whether the large number of gaze variables may be captured by distinct dimensions based on empirical patterns (i.e., principal components), representing most of the information from the original variables (81). Variables in the PCA included: standard gaze variables (dwell time, fixation count), fixation patterns (perseverative fixations, regressive fixations, fixation transition analyses), first fixation duration, and distribution patterns (fixation rates, fixation spatial distribution/coverage analyses) (see asterisks in Table 2). All data from proband and parent groups were included to generate components. Regression factor scores were generated for each component for each individual, and analyzed using one-way ANOVAs to examine group differences among the proband and parent/BAP groups and controls.

\section{Results}


Detailed statistical analyses are reported in Tables 3 and 4. 
Table 3

Summary of Results - ASD versus control groups

\section{Control ASD Group Differences \\ Group Group}

$\mathrm{M}(\mathrm{SD}) \quad \mathrm{M}(\mathrm{SD}) \quad F \quad$ Pillai's $d f \quad p$

Trace

partial

$\eta^{2}$

\section{Standard Gaze Variables}

(1) Dwell time (\%) - social

75.54

(12.41)

70.37

(16.21)

$1.40 \quad 0.05$

2

0.255

0.050

(1) Dwell time (\%) - non-social

$14.54 \quad 19.03$

(7.32)

(12.63)

(2) Fixation count (\%) - social

71.25

(12.63)

67.46

(15.74)

$0.65 \quad 0.02$

$2, \quad 0.524$

0.024

(2) Fixation count (\%) - non-social

$17.85 \quad 21$

(7.98) (13.42)

\section{Fixation Patterns}

(4) Perseverative fixations (\%) social*

(4) Perseverative fixations (\%) non-social*

(5) Regressive fixations (\%) - social

(5) Regressive fixations (\%) - nonsocial

(6) Fixation transition

Social to social (\%)

Non-social to non-social (\%)

Non-social to non-social (\%)

Social to non-social (\%)

Non-social to social (\%)
$3.27 \quad 0.11$

(30.88) (35.51)

23.4

(32.13)

7.08

(14.94)

71.01

(12.43)

67.22

(16.43)

$\begin{array}{ll}0.62 & 0.02\end{array}$

21.3

(8.77)

(13.54)

53 


\section{Distribution patterns}

(9) Fixation rate (exploration)

(10) Fixation rate (exploration) AOI

(fix/s) - social

(10) Fixation rate (exploration) AOI

(fix/s) - non-social

(11) Fixation spatial

distribution/coverage $-5 \times 4$

(larger)

(11) Fixation spatial

distribution/coverage - $10 \times 8$

(smaller)
3.34

(.56)

3.10

(0.59)

3.52

$(.60)$

3.37

$(0.7)$

$1.51 \quad 0.06$

1.35

$\begin{array}{lll}1, & 0.251 & 0.024\end{array}$

54

$\begin{array}{lll}2, & 0.231 & 0.055\end{array}$

$4.33 \quad 4.09$

(1.05)

(1.47)

$35.32 \quad 35.97$

(5.29)

(6.62)

$0.17 \quad-$

$54 \quad 0.686 \quad 0.003$

62.92

(13.59)

$2.14 \quad-$

$1, \quad 0.149$

0.038

54

Bold values indicate significance at $p<.05$; *Non-parametric Mann-Whitney U results are presented in the body of the manuscript; $F E T=$ Fisher's Exact Test 
Table 4

Summary of Results - ASD parent versus control parent groups

$\begin{array}{lll}\text { Control } & \text { ASD } & \text { Group Differences } \\ \text { Parent } & \text { Parent } & \\ \text { Group } & \text { Group } & \end{array}$

$M(S D) \quad M(S D) \quad F \quad \begin{array}{lllll}\text { Pillai's } & d f & p & \substack{\text { partial } \\ \eta^{2}}\end{array}$

\section{Standard Gaze Variables}

(1) Dwell time (\%) - social

(1) Dwell time (\%) - non-social

(2) Fixation count (\%) - social

(2) Fixation count (\%) - nonsocial
71.57

(13.95)

18.87

(10.05)

68.17

(13.27)

21.28

(9.33)
65.5

(11.67)

22.36

(8.76)

63.71

(10.24)

$1.98 \quad 0.04$

2

0.144

2,

0.066

0.055

24.38

(8.46)

\section{Fixation Patterns}

(4) Perseverative fixations (\%) social*

(4) Perseverative fixations (\%) non-social*

(5) Regressive fixations (\%) social

(5) Regressive fixations (\%) non-social
72.83

(30.64)

17.89

(23.93)

68.5

(13.69)

21.5

(9.61)
62.8

(33.78)

$\begin{array}{ll}1.50 & 0.03\end{array}$

$2, \quad 0.228$

97

18

(22.19)

63.44

(10.44)

$2.31 \quad 0.05$

24.8

(8.92)

(6) Fixation transition

$\begin{array}{lll}\text { Social to social (\%) } & 58.37 & 53.45 \\ & (16.46) & (14.13) \\ \text { Non-social to non-social (\%) } & 6.84 & 8.95 \\ & (7.77) & (7.95) \\ \text { Social to non-social (\%) } & 17.70 & 18.75 \\ & (7.42) & (7.32) \\ \text { Non-social to social (\%) } & 17.09 & 18.85 \\ & (7.52) & (7.28)\end{array}$

First Fixations

FET

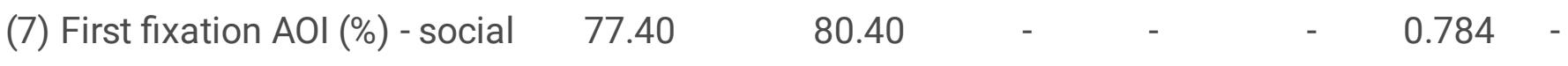

(7) First fixation AOI (\%) - non- $\quad 22.60 \quad 19.60$ social 


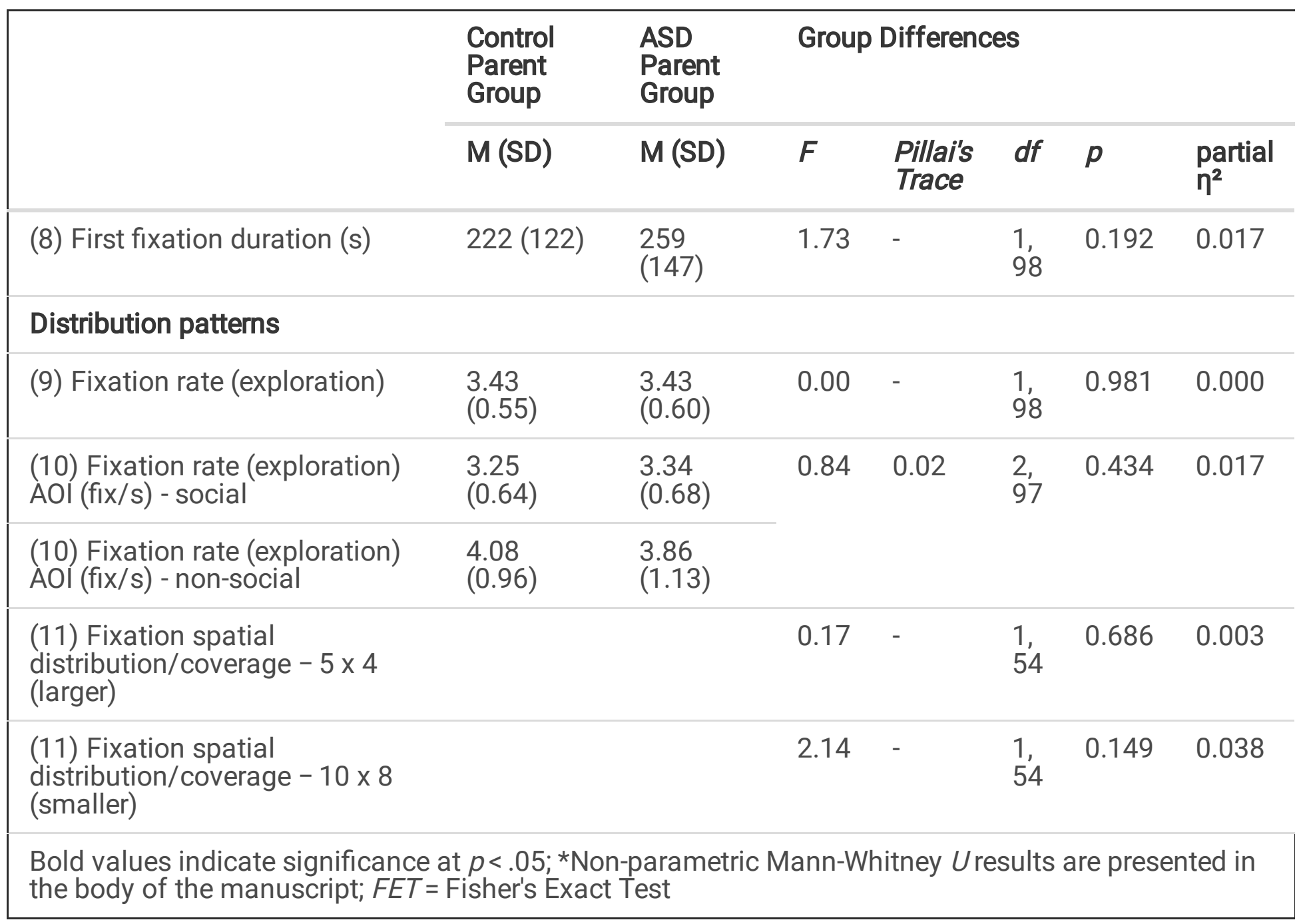

Standard gaze variables (Fig. 3)

Findings from the standard gaze variables have been previously reported with different statistical analyses (Lee et al., 2019) and are reported below for the purpose of comparison to non-standard analytic methods.

(1) Dwell time:

Probands. There were no significant differences between the ASD and control group in the percentage of time spent attending towards social or non-social information $\left(\mathrm{F}_{(2,53)}=1.40\right.$, Pillai's Trace $=.05, p=.255$, partial $\left.\eta^{2}=.050\right)$.

Parents. No significant differences emerged in the multivariate test between parents across social and non-social looking $\left(\mathrm{F}_{(2,97)}=2.80\right.$, Pillai's Trace $=.06, p=.066$, partial $\left.\eta^{2}=.055\right)$.

BAP. The overall model for social and non-social looking times was significant across BAP(+), BAP(-), and control parents $\left(F_{(4,197)}=2.71\right.$, Pillai's Trace $=.11, p<.05$, partial $\left.\eta^{2}=.053\right)$, with significant differences emerging in both social $\left(\mathrm{F}_{(2,97)}=3.70, p<.05\right.$, partial $\left.\eta^{2}=.071\right)$ and non-social $\left(\mathrm{F}_{(2,97)}=4.44, p<.05\right.$, partial $\eta^{2}=.084$ ) looking time. Pairwise comparisons revealed that $\mathrm{BAP}(+)$ parents showed significantly 
reduced social looking compared to the control parent group (mean difference $=-8.77, p<.01$ ) and increased non-social looking compared to both BAP(-) parents (mean difference $=5.51, p<.05$ ) and control parents (mean difference $=6.84, p<.05$ ).

(2) Fixation count:

Probands. No significant group differences emerged in the percentage of fixations directed towards social or non-social information $\left(\mathrm{F}_{(2,53)}=.65\right.$, Pillai's Trace $=.02, p=.524$, partial $\left.\eta^{2}=.024\right)$.

Parents. Similarly, no significant differences emerged between parent groups $\left(\mathrm{F}_{(2,97)}=1.98\right.$, Pillai's Trace $=.04, p=.144$, partial $\eta^{2}=.039$ ).

BAP. No significant differences in BAP status emerged overall for social or non-social looking $\left(F_{(4,194)}=\right.$ 1.42 , Pillai's Trace $=.06, p=.229$, partial $\eta^{2}=.028$ ).

Temporal Dynamics (Fig. 4)

(3) Fixations over time (Growth Curve Analyses; Table 5, Fig. 4): 
Table 5

Summary of Results for GCA analyses - Fixations over time

\begin{tabular}{|c|c|c|c|c|c|c|c|c|c|}
\hline & \multicolumn{9}{|c|}{ Control > ASD } \\
\hline & Estimate & $t$ & \multicolumn{7}{|l|}{$p$} \\
\hline Intercept & -0.74 & -30.42 & \multicolumn{7}{|l|}{$<.0001$} \\
\hline Linear & -0.10 & -0.75 & \multicolumn{7}{|l|}{0.453} \\
\hline Quadratic & -0.21 & -1.58 & \multicolumn{7}{|l|}{0.120} \\
\hline \multirow[t]{3}{*}{ Cubic } & -0.01 & -0.05 & \multicolumn{7}{|l|}{0.962} \\
\hline & \multicolumn{9}{|c|}{ Control parent > ASD parent } \\
\hline & Estimate & $t$ & \multicolumn{7}{|l|}{$p$} \\
\hline Intercept & -0.72 & -44.73 & \multicolumn{7}{|l|}{$<.0001$} \\
\hline Linear & 0.16 & 1.53 & \multicolumn{7}{|l|}{0.126} \\
\hline Quadratic & 0.10 & 0.92 & \multicolumn{7}{|l|}{0.360} \\
\hline \multirow[t]{3}{*}{ Cubic } & 0.23 & 2.24 & \multicolumn{7}{|l|}{0.025} \\
\hline & \multicolumn{3}{|c|}{ Control parent > BAP(+) } & \multicolumn{3}{|c|}{ Control parent > BAP(-) } & \multicolumn{3}{|c|}{$\mathrm{BAP}(-)>\mathrm{BAP}(+)$} \\
\hline & Estimate & $t$ & $p$ & Estimate & $t$ & $p$ & Estimate & $t$ & $p$ \\
\hline Intercept & -0.08 & -0.26 & 0.010 & -0.15 & -0.54 & 0.590 & -0.07 & -2.08 & 0.038 \\
\hline Linear & 0.44 & 3.31 & 0.001 & -0.02 & -0.16 & 0.876 & 0.46 & 3.38 & 0.001 \\
\hline Quadratic & 0.39 & 2.91 & 0.004 & -0.09 & -0.76 & 0.446 & 0.48 & 3.50 & 0.001 \\
\hline Cubic & 0.42 & 3.20 & 0.001 & 0.11 & 0.10 & 0.320 & 0.30 & 2.28 & 0.023 \\
\hline
\end{tabular}

Probands. There were no significant group differences detected across linear (Estimate $=.10, t(832)=.75$, $p=.453)$, quadratic $($ Estimate $=.21, t(832)=1.58, p=.12)$, or cubic $($ Estimate $=.006, t(832)=.05, p=.962)$ terms in social versus non-social looking patterns over the course of the stimulus presentation. Time-bin divergence analyses revealed significant differences occurring halfway through the stimulus presentation, where individuals with ASD demonstrated significantly reduced social looking between 3000 and 3300 ms compared to controls. These findings indicate that individuals with ASD disengage from social information early, decreasing social fixations over time, whereas the control group showed increased social looking initially, that also decreased over the course of the task.

Parents. In contrast to findings in the ASD group, a significant group difference was detected for the cubic polynomial term $($ Estimate $=-.23, t(1474)=-2.24, p<.05)$, indicating that the ASD parent group shifted away earlier from social AOIs, and demonstrated decreased attention to social information over time, 
compared to the control parent group. To examine the exact moments at which time points groups differed from one another, divergence analyses demonstrated that ASD parents demonstrated reduced social looking patterns primarily during the beginning (i.e., 300-600 ms) and end (i.e., 4500-4800 ms and 6900-7200 ms) of the stimulus presentation (see Fig. 4). Interestingly, ASD parents also demonstrated increased social looking relative to the control parent group between 5100-5400 ms, showing a dynamic pattern of an increase followed by a decrease in social looking towards the end of the stimulus presentation, compared to the control parent group.

BAP. Significant group differences were detected for the linear, quadratic, and cubic terms, indicating that $\mathrm{BAP}(+)$ parents shifted away from social stimuli earlier, and demonstrated decreased social attention over the course of the task compared to the BAP(-) (linear estimate $=.46, t(1474)=3.38, p<.001$; quadratic estimate $=.48, t(1474)=3.50, p<.001$, cubic estimate $=.30, t(1474)=2.28, p<.05)$ and control parent groups (linear estimate $=.44, t(1474)=3.31, p<.001$; quadratic estimate $=.39, t(1474)=2.91, p<.001$, cubic estimate $=.42, t(1474)=3.20, p=.001)$. BAP(-) and control parent groups did not differ from one another across any linear, quadratic, or cubic terms (linear estimate $=-.02, t(1474)=-.16, p=.876$; quadratic estimate $=-.09, t(1474)=-.76, p=.446$, cubic estimate $=.11, t(1474)=.10, p=.320)$.

Divergence tests showed that the $\mathrm{BAP}(+)$ group fixated more towards social information relative to the control parent group towards the second half of the stimulus presentation (i.e., $5100-5400 \mathrm{~ms}$ ), and showed a stark decrease towards the end of the viewing window (i.e., 6900-7200 ms). Similarly, the $\mathrm{BAP}(+)$ group showed significantly decreased social attention towards the end of the stimulus compared to the BAP(-) parent group between 6900-7200 ms. In contrast, the BAP(-) group showed reduced social attention towards the second half of the stimulus presentation (i.e., $4500-4800 \mathrm{~ms}$ ), with a later (i.e., 5100-5400) increase in social attention relative to controls.

\section{Fixation patterns (Fig. 5-7)}

(4) Perseverative fixations:

Probands. MANOVA results revealed significant differences between ASD and control groups in the percentage of perseverative fixations towards social and non-social information $\left(F_{(2,53)}=3.27\right.$, Pillai's Trace $=.11, p<.05$, partial $\eta^{2}=.110$ ). Follow-up univariate ANOVA tests revealed that the ASD group made a greater proportion of perseverations on non-social information compared to the control group $\left(\mathrm{F}_{(1,54)}=\right.$ $6.43, p<.05$, partial $\left.\eta^{2}=.106\right)$. Follow-up non-parametric analyses confirmed these findings, $A S D \mathrm{M}_{\text {rank }}=$ 32.98; controls $\mathrm{M}_{\text {rank }}=25.14 ; U=276.5, p<.05$. No significant differences emerged between groups for social perseverations $\left(A S D \mathrm{M}_{\text {rank }}=26.13\right.$, Control $\left.\mathrm{M}_{\text {rank }}=30.28, U=327, p=.324\right)$.

Parents. No significant group differences were found between ASD parent and control parent groups $\left(F_{(2,97)}=1.50\right.$, Pillai's Trace $=.03, p=.228$, partial $\left.\eta^{2}=.030\right)$. Non-parametric analyses confirmed these findings (social AOI: $A S D$ parent $\mathrm{M}_{\text {rank }}=47.01$, Control parent $\mathrm{M}_{\text {rank }}=55.96, U=976.5, p=.122$; non-social AOI: $A S D$ parent $\mathrm{M}_{\text {rank }}=50.97$, Control parent $\mathrm{M}_{\text {rank }}=49.77, U=1161, p=.827$ ). 
BAP. MANOVA results showed no difference in social or non-social perseverative looking patterns between $\operatorname{BAP}(+)$, BAP( $(-)$, or control parent groups $\left(\mathrm{F}_{(4,194)}=1.59\right.$, Pillai's Trace $=.06, p=.179$, partial $\eta^{2}=$ .032). However, non-parametric Mann-Whitney $U$ test revealed that the $B A P(+)$ group $\left(M_{\text {rank }}=26.31\right)$ made significantly fewer perseverations towards social information compared to the control parent group $\left(M_{\text {rank }}\right.$ $=35.50)(U=331.5, p<.05)$.

(5) Regressive fixations:

Probands. No significant group differences emerged for social and non-social regressive fixations $\left(\mathrm{F}_{(2,53)}\right.$ $=.62$, Pillai's Trace $=.02, p=.541$, partial $\eta^{2}=.023$ ).

Parents. Parent groups also showed comparable social and non-social regressive fixations $\left(F_{(2,97)}=2.31\right.$, Pillai's Trace $=.05, p=.104$, partial $\left.\eta^{2}=.046\right)$.

BAP. No significant differences in social and non-social regressive fixations emerged by BAP status $\left(F_{(4,194)}=1.87\right.$, Pillai's Trace $=.07, p=.118$, partial $\left.\eta^{2}=.037\right)$.

(6) Fixation transition analyses:

Probands. There were no significant differences in the percentage of transitions occurring (regardless of direction of fixation transitions) between social and non-social information $\left(F_{(3,52)}=.98\right.$, Pillai's Trace $=.05, p=.410$, partial $\left.\eta^{2}=.053\right)$.

Parents. Likewise, parents showed no differences in the percentage of transitions made between social and non-social stimuli $\left(F_{(3,96)}=1.02\right.$, Pillai's Trace $=.03, p=.387$, partial $\left.\eta^{2}=.031\right)$.

BAP. BAP status did not affect overall findings across specific transition patterns $\left(F_{(6,192)}=.72\right.$, Pillai's Trace $=.04, p=.637$, partial $\eta^{2}=.022$ ).

First fixations.

(7) First fixation AOI:

Probands. Fisher's Exact test revealed no significant differences in first fixations towards social ( $A S D 39 \%$, Control 49\%) and non-social information (ASD 4\%, Contro/ 8\%) between groups ( $p=.688$ ).

Parents. Similarly, no significant differences emerged between first fixation looks to social (ASD parent $50 \%$, Control parent $29 \%$ ) versus non-social (ASD parent $12 \%$, Control parent $9 \%$ ) information between parent groups $(p=.784)$.

BAP. Similarly, no differences emerged when considering BAP status in parents in the proportion of social $(B A P(+) 21 \%, B A P(-) 29 \%$, control parent $29 \%)$ and non-social $(B A P(+) 2 \%, B A P(-) 10 \%$, Control parent $9 \%)$ first fixations. 
(8) First fixation duration:

Probands. The ASD group did not differ from the control group in the duration of their first fixations $\left(\mathrm{F}_{(1,54)}=.793, p=.377\right.$, partial $\left.\eta^{2}=.014\right)$.

Parents. There were no significant group differences in the time spent initially fixating on the scene ( $F_{(1,98)}=1.73, p=.192$, partial $\left.\eta^{2}=.017\right)$.

BAP. Similarly, there were no significant group differences between BAP(+), BAP(-), or control parent groups $\left(\mathrm{F}_{(2,97)}=.85, p=.429\right.$, partial $\left.\eta^{2}=.017\right)$ in the time spent first fixating on the scene. .

Distribution patterns (Fig. 8)

(9) Fixation rate (exploration):

Probands. The ASD and control groups demonstrated a similar number of fixations per second of track time regardless of $\mathrm{AOI}\left(\mathrm{F}_{(1,54)}=1.35, p=.251\right.$, partial $\left.\eta^{2}=.024\right)$.

Parents. Similarly, control parents showed comparable exploration across the scene $\left(F_{(1,98)}=.001, p\right.$ $=.981$, partial $\left.\eta^{2}<.0001\right)$.

BAP. There were no differences across BAP groups and controls $\left(\mathrm{F}_{(2,97)}=.17, p=.848\right.$, partial $\left.\eta^{2}=.003\right)$.

(10) Fixation rate (exploration) AOI:

Probands. ASD and control groups did not differ significantly in the number of fixations per second of track time made towards social and non-social information $\left(\mathrm{F}_{(2,52)}=1.51\right.$, Pillai's Trace $=.06, p=.231$, partial $\left.\eta^{2}=.055\right)$.

Parents. Similarly, parents did not show group differences in exploration towards social and non-social AOls $\left(\mathrm{F}_{(2,97)}=.84\right.$, Pillai's Trace $=.02, p=.434$, partial $\left.\eta^{2}=.017\right)$.

BAP. Similarly, parent findings remained non-significant regardless of BAP status $\left(F_{(4,194)}=1.794\right.$, Pillai's Trace $=.07, p=.132$, partial $\left.\eta^{2}=.036\right)$.

(11) Fixation spatial distribution/coverage:

Probands. There were no significant differences between the ASD and control groups in the percentage of unique areas explored during the matrix for larger $\left(\mathrm{F}_{(1,54)}=.17, p=.686\right.$, partial $\left.\eta^{2}=.003\right)$ or smaller $\left(\mathrm{F}_{(1,54)}=2.14, p=.149\right.$, partial $\left.\eta^{2}=.038\right)$ "boxes".

Parents. Similarly, parents showed comparable area coverage across the larger $\left(\mathrm{F}_{(1,98)}=2.96, p=.088\right.$, partial $\left.\eta^{2}=.029\right)$ and smaller $\left(F_{(1,98)}=2.39, p=.126\right.$, partial $\left.\eta^{2}=.024\right)$ grid matrices. 
BAP. There were also no differences between $\mathrm{BAP}(+), \mathrm{BAP}$, and control parent groups in the percentage of fixations covering the scene when the matrix was comprised of large $\left(\mathrm{F}_{(2,97)}=1.52, p=.225\right.$, partial $\eta^{2}=$ $.030)$ or small $\left(\mathrm{F}_{(2,97)}=1.29, p=.281\right.$, partial $\left.\eta^{2}=.026\right)$ "boxes".

\section{Principal Component Analysis (PCA)}

Examination of the scree plot (Supplementary Fig. 1) led to a two-component solution describing $58.6 \%$ of the variance of the data. The PCA yielded adequate loadings $(\geq 0.30)$ on the two components (Supplementary Table 1). The first component consisted of all fixation variables tapping social versus non-social looking (henceforth, "social/non-social attention factor". The second component consisted of fixation rates versus fixation spatial coverage efficiency-essentially reflecting the overall efficiency of spatial exploration in terms of fixation rates (henceforth, "efficiency of exploration"). For example, an individual scoring high on this component would have fixations that covered a relatively greater area, even if occurring less frequently; or conversely, fixations that were spatially confined even given an increased rate of fixation.

Probands. There were no significant group differences between the ASD and control groups on the social/non-social attention factor $\left(\mathrm{F}_{(1,53)}=2.39, p=.128\right.$, partial $\left.\eta^{2}=.043\right)$ or on the efficiency of exploration factor $\left(\mathrm{F}_{(1,53)}=.05, p=.818\right.$, partial $\left.\eta^{2}=.001\right)$.

Parents. The ASD parent group demonstrated significantly lower scores on the social/non-social attention factor (lower value indicating less social and more non-social looking) compared to the control parent group $\left(F_{(1,95)}=4.40, p<.05\right.$, partial $\left.\eta^{2}=.044\right)$. No differences in efficiency of exploration factor scores emerged between groups $\left(\mathrm{F}_{(1,95)}=.33, p=.568\right.$, partial $\left.\eta^{2}=.003\right)$.

BAP. Overall, there was a significant effect for the BAP $\left(F_{(2,97)}=3.29, p<.05\right.$, partial $\left.\eta^{2}=.064\right)$, with the $\mathrm{BAP}(+)$ group scoring significantly lower on the social/non-social attention factor relative to the control parent group (mean difference $=.582, p=.01$ ). No significant differences were found in efficiency of exploration factor scores by BAP status $\left(\mathrm{F}_{(2,97)}=.13, p=.880\right.$, partial $\left.\eta^{2}=.003\right)$.

Table 6: Summary of Results - Group differences in factor scores derived from the PCA 


\begin{tabular}{|c|c|c|c|c|c|c|}
\hline & $M(S D)$ & $M(S D)$ & $F$ & $d f$ & $p$ & $\begin{array}{l}\text { partial } \\
\eta^{2}\end{array}$ \\
\hline & Control Group & ASD Group & & & & \\
\hline $\begin{array}{l}\text { Social/non-social attention } \\
\text { (factor } 1 \text { ) }\end{array}$ & $0.45(0.88)$ & $-0.02(1.38)$ & 2.39 & $\begin{array}{l}1 \\
53\end{array}$ & 0.128 & 0.043 \\
\hline \multirow[t]{2}{*}{$\begin{array}{l}\text { Efficiency of exploration (factor } \\
\text { 2) }\end{array}$} & $0.08(1.01)$ & $0.01(1.12)$ & 0.06 & $\begin{array}{l}1 \\
53\end{array}$ & 0.818 & 0.001 \\
\hline & $\begin{array}{l}\text { Control Parent } \\
\text { Group }\end{array}$ & $\begin{array}{l}\text { ASD Parent } \\
\text { Group }\end{array}$ & & & & \\
\hline $\begin{array}{l}\text { Social/non-social attention } \\
\text { (factor } 1 \text { ) }\end{array}$ & $0.10(1.01)$ & $-0.29(0.81)$ & 4.40 & $\begin{array}{l}1, \\
95\end{array}$ & 0.039 & 0.044 \\
\hline $\begin{array}{l}\text { Efficiency of exploration (factor } \\
\text { 2) }\end{array}$ & $-0.08(0.85)$ & $0.04(1.06)$ & 0.33 & $\begin{array}{l}1, \\
95\end{array}$ & 0.568 & 0.003 \\
\hline $\begin{array}{l}\text { Bold values indicate } \\
\text { significance at } p<.05\end{array}$ & & & & & & \\
\hline
\end{tabular}

\section{Discussion}

This study applied a suite of eye-tracking analyses in attempt to deeply characterize potential differences in visual attention patterns to social and non-social information in ASD and the broad autism phenotype (BAP). Relatively robust differences were observed in patterns of fixations over the course of the stimulus presentation in the ASD and BAP parent groups, where both groups decreased social attention over time. Both the ASD and BAP(+) parent groups also showed differences in repeat perseverative fixations, with increased non-social and decreased social visual perseveration, respectively. Notably, although additional eye tracking analyses revealed relatively few differences in ASD and parent groups (regardless of BAP status), reducing data through principal component analysis revealed more robust group differences among parents, and these appeared largely driven by the subgroup showing the BAP. These findings underscore the utility of applying a broad array of analytic approaches to capture what may be nuanced, but still highly meaningful differences in visual attention in ASD and clinically unaffected relatives.

As noted, standard gaze variables (i.e., percentage of fixation duration and percentage of fixations) were re-produced from prior work (23) for comparison with the more expansive battery of variables examined here, and showed that parents with BAP traits spent more time generally fixating on non-social information compared to both parents without BAP features and control parents. Individuals with ASD in prior work showed similar trending patterns (23). Gaze analyses beyond these standard variables were unique to the present study, with growth curve analyses examining changes in visual attention over time emerging as a primary variable distinguishing the ASD and BAP(+) groups from controls and BAP(-) parents. For these groups, fixations towards social information changed over the course of the task, wherein ASD and BAP(+) groups showed distinct fixation patterns from controls. Parents with the BAP shifted away earlier from social information, and showed decreased social attention over time compared 
to parents without the BAP and control parents. While this overall pattern was not significantly different in the ASD group, examination of the data revealed qualitatively similar fixation patterns over the course of the stimulus presentation in ASD and the BAP. Importantly, divergence analyses, performed to determine the exact moments in which groups diverged from each other, demonstrated that individuals with ASD showed significantly reduced social attention towards the middle of the stimulus presentation compared to controls, while $\mathrm{BAP}(+)$ parents showed a later divergence from control parents, occurring towards the second half, and an even more dramatic decline in attention to social AOls in the final seconds of the stimulus presentation, compared to both control parents and BAP(-) parents. It therefore appears necessary to examine timing effects in studies of visual perception and attention, particularly given prior work evidencing delayed global (i.e., gestalt or integrative) processing in non-social tasks in ASD (82) and atypicalities in the underlying neural correlates indexing face perception in later time windows $(83,84)$. It is possible that in more dynamic stimuli involving prolonged looking, findings of decreased social attention may result in information overload (e.g., see review 85). Finally, results highlight how standard gaze variables may obscure shifting patterns of attentional engagement documented here, which may be meaningful to consider in understanding social-emotional processing differences in ASD. As such, an examination of fixations over the course of a stimulus presentation and divergence over specific time bins becomes an important step in further disentangling the nuanced and dynamic nature of gaze inherent to human behavior and impacted in ASD.

A prior study with this same sample, examining only standard eye-tracking variables (23) concluded that increased attention allocation towards non-social information in the TAT scene may reflect greater cognitive effort required to support narrative production (given the nature of the task); however, this pattern of visual attention did not improve narrative quality, suggesting that groups capitalized on different sources of information to inform their narratives. Patterns revealed by the more extensive gaze analyses performed in the current study suggest that these standard variables may not be sufficiently sensitive to capturing important shifts in attention during the narrative task. Specifically, increased attention towards non-social information over time suggests that shifting attention towards non-salient aspects of a scene may be advantageous in gleaning meaning from a scene to inform meaningful narratives. Given these findings, it is therefore possible that an examination of the second half of the scene (where individuals with ASD and ASD parents have already disengaged from social stimuli), may align more consistently with prior work that showed relationships between increased attention to nonsalient information and greater clinical-behavioral impairments (40). For example, individuals with ASD have been observed to demonstrate reduced attention towards the eye region of the face and more attention towards the mouth (17) and, during natural scenes in this same study, increased fixations towards non-social information was associated with poorer social adjustment and increased ASD symptom severity (17), complementing findings from a later study that showed relationships between elevated fixations directed towards the background of a scene that related to increased ASD symptom severity (36). Future studies might benefit from a step-wise method of analysis that first includes an examination of fixations over the course of a task via GCA, followed by an application of divergence time bin analyses, and subsequent assessment of traditional and unique gaze analytical tools applied in the 
present study during critical time windows showing divergent patterns only. Together, that individuals with ASD and the BAP showed decreased fixations directed towards social information over time, suggests that this looking pattern may be particularly sensitive in reflecting ASD genetic vulnerability.

Other eye-tracking indices beyond standard fixation duration and proportion of fixations revealed further differences associate with ASD and the BAP. Specifically, individuals with ASD showed elevated rates of perseverative fixations towards non-social information and parents with the BAP showed reduced perseverations towards social AOls. It may be that perseverative fixations reflect rigid tendencies, or a tendency to visually get "stuck" on certain visual information (55). Prior work has also documented such patterns of perseveration among individuals with ASD or the BAP during eye-tracking tasks involving social and non-social images $(28,86)$, complex scenes $(87)$, and language processing $(27,88)$. Such perseverative tendencies are manifested behaviorally and are among the defining characteristics of ASD. They may also be evident in more subtle forms in the BAP (89-91), revealed through rigid or inflexible personality traits and behaviors reported in every-day life through both self-report questionnaires and objective, semi-structured interviews $(49,50,64,92-95)$.

Although relationships between gaze variables and clinical-behavioral features are not entirely consistent $(17,23,36,40)$, such patterns of refixations (i.e., perseverative or regressive fixations) have been shown to relate to both lower-order motoric RRBs and social communication in $\operatorname{ASD}(27,28)$ and the BAP in parents (27). A study examining gaze-language coordination additionally identified specific associations emerging between refixations in parents with the BAP and elevated rates of RRBs in their children (27). Findings of atypical perseverative visual attention documented in ASD and the BAP in the present study may suggest that perseverative fixations or "sticky" visual attention could inform patterns of inheritance of ASD-related candidate endophenotypes. It additionally highlights the utility of examining eye-tracking data using methods beyond traditional fixation and duration eye-tracking variables.

Finally, it is possible that increased perseverative fixations towards non-social information in ASD and decreased preservative fixations towards social information may stem from differences in local (i.e., detailed) and global (i.e., integrative) visual processing in ASD more broadly $(82,96)$. Individuals with ASD have been shown to demonstrate heightened local perceptual abilities (i.e., the enhanced perceptual functioning theory) which may result in a reduction in global perceptual abilities (i.e., weak central coherence theory), suggesting that they have difficulty shifting attention from local to a global level (97, 98). Enhanced local perceptual abilities also may explain the tendency for individuals with ASD to more often focus on or get distracted by insignificant, non-salient details in their environment, which has been documented in both eye-tracking studies $(23,40)$ and autobiographical accounts (e.g., 99). As such, it is possible that the findings documented in the present study contribute to both the social deficit and weak central coherence/enhanced perceptual functioning theories of ASD, which further necessitates studies examining the links between these theories.

In contrast to these relatively robust differences noted in perseverative fixations, there were few differences in proband and parent groups when applying other eye-tracking analytical techniques. 
Specifically, first fixation AOI or duration did not differ between groups. This was somewhat surprising, particularly in light of social orientation differences reported repeatedly in $\operatorname{ASD}(20,37,100)$. It is possible that the demands of the task (i.e., providing a narrative after viewing the picture) influenced visual attention patterns in ways distinct from the passive viewing tasks used in much of prior work, where participants may explore the image as they please $(28,101)$. Moreover, given that prior work has shown a lack of social orientation differences in higher functioning individuals with ASD (e.g., 102), the higher cognitive ability of the sample included in the present study (i.e., verbal IQ > 80) may have further contributed to the null findings. First fixation methods may thus be more appropriate during infancy when social orientation comes online, applied to individuals who may have lower IQ, task methods involving passive viewing, and/or more dynamic stimuli.

Similarly, the lack of differences in fixation rate/exploration (i.e., number of fixations per second), transition, or fixation spatial distribution/fixation coverage analyses were surprising, given that prior work has documented differences in ASD, particularly during a face processing task (60). As such, it may be that the concurrent narrative task demands necessitated both individuals with ASD and controls to explore the complex scene generously and comparably to deduce information to help inform their narratives; this stands in contrast to stimuli depicting motionless faces given their reduced "clutter" and visual complexity. Indeed, there is strong evidence in ASD demonstrating that patterns of fixations vary and depend highly on the context (40). As such, use of fixation transitions, coverage, and exploration may be applied to future work examining visual attentional differences in the BAP during a face processing task, particularly given distinct face processing emerging in parents that have also been linked to underlying neural correlates (13).

Despite there being no group differences on most of these individual variables outlined above, when considered together, using data reduction methods to identify variables tapping similar constructs, they were fruitful in increasing power to detect subclinical differences associated with the BAP. Specifically, robust BAP-specific differences were observed on the social/non-social factor score compared to control parents, indicating that parents of individuals with the BAP looked significantly less at social information than ASD parents without the BAP or controls. Although significant group differences in scores of social/non-social looking were not identified between the ASD and control groups, the effect size of the difference was medium (and comparable to that of the parent groups). Of note, distribution measures that did not take social/non-social differences into account did not differentiate between parent or proband groups, underscoring the specificity of social communicative differences among ASD and the BAP, and providing further evidence that social attention differences may serve as a strong candidate as an ASD endophenotype. These findings also emphasize the utility of extracting multiple eye-tracking outcome variables and synthesizing them through data reduction methodologies, such as PCA, such that the emergent properties associated with mechanistic underpinnings of special populations can be decoded. It is important to note that the results generated from PCA required the assembly of a large number of potential candidate eye-tracking markers associated with ASD risk, and that the interpretation and understanding of the specificity of the constructs required an in depth, independent examination of each marker's specific performance and properties. This work may serve to capture subtle phenotypic 
variability associated with social-communicative impairments in these groups, and perhaps lead to more powerful, objective tools in monitoring treatment outcome. Taken together, findings from this study may further our understanding of eye-tracking methods, their application to special populations and associated characterization of endophenotypes, and underlying cognitive and biological mechanisms contributing to ASD and the BAP.

\section{Limitations}

Although adequately powered, this study included a relatively small sample size of individuals with ASD and controls, and larger samples may be useful in further exploring ASD-related gaze differences, particularly for the principal component analysis. This study also applied eye-tracking analyses to only one context (i.e., a complex scene depicting both social and non-social information). Future studies are warranted to further examine how these unique eye-tracking methods may be applied to studies of the BAP across stimuli varying in context. It is also important to acknowledge that the 11 methodological tools documented here, while applicable to research in ASD and subclinical features related to ASD, represent only a subset of a large number of analytic measures that can be applied to eye-tracking studies (e.g., see 103).

\section{Conclusions}

Taken together, this study highlights the specific utility of growth curve analyses to characterize meaningful fixation differences over the course of a task studies of visual social attention in ASD and the BAP. By providing a detailed examination of looking patterns over time, growth curve analyses may better capture the dynamic aspect of gaze that typically occurs in natural settings and which are impacted in ASD. In contrast, average dwell time and proportion of fixation variables assume a uniform or stagnant method of exploration, and tend to attenuate potential differences in looking patterns. Additionally, perseverations may be specifically tied to greater ASD risk, given differences observed in both ASD and the BAP. Despite most other variables yielding no robust differences between groups independently, when considered together using principal component analysis, this broad suite of eye-tracking variables contributed critical information to revealing relatively striking differences in social attention between the BAP and ASD from respective control groups. The eye-tracking variables examined in this study are thought to effectively reveal different aspects of underlying cognition $(1,9,55-59,77)$, and therefore may reveal key mechanistic insights into the roots of social functioning differences in ASD. These methods may additionally benefit studies of social skills training in individuals with ASD, as an objective means of measuring treatment outcomes. To our knowledge, this study is the most comprehensive application of different types of eye-tracking analytical methods in ASD and the BAP to date. Given the objective and measurable nature of the rigorous eye-tracking variables documented here, findings might contribute to development of a template that could be drawn upon in future eye-tracking studies examining visual attention across ASD and other populations. 


\section{Abbreviations}

$\mathrm{AOI}$

Area of interest

ASD

Autism spectrum disorder

BAP

Broad autism phenotype

MANOVA

multivariate analysis of variance

TAT

Thematic Apperception Test

\section{Declarations}

Ethics approval and consent to participate. Ethical approval. All procedures performed in this study were in accordance with the ethical standards of the institutional research committee and with the 1964 Helsinki declaration and its later amendments or comparable ethical standards. Informed consent. Informed consent was obtained from all individual participants included in the study.

Consent for publication. Not applicable

Availability of data and materials. The datasets used and/or analyzed during the current study are available from the corresponding author on reasonable request.

Competing interests. FS is a paid consultant for Roche and Janssen pharmaceutical companies. All other authors declare that they have no disclosures or conflict of interest.

Funding. This research was supported by grants from the National Institutes of Health (R01DC010191, R01MH091131) and the National Science Foundation (BCS-0820394) and P30 HD03110.

Authors' contributions. ML conceived the study from which data were drawn, contributed to the development of the study design and goals, and oversaw data collection, analyses, and interpretation and writing of the manuscript. KN conceived the study and assisted with data acquisition, led processing of data, the development of eye-tracking variables, data analyses, and manuscript preparation. FS assisted with data processing, conception of analytic methods, interpretation, and manuscript preparation. MW assisted with acquisition of data, data processing, and contributed to manuscript preparation. All authors read and approved the final manuscript.

Acknowledgments. We are grateful to the individuals and families who participated in this study. We are additionally grateful to Nell Maltman, Ph.D. and Michelle Lee, Ph.D. for their contributions to data processing. 


\section{References}

1. Eckstein MK, Guerra-Carrillo B, Singley ATM, Bunge SA. Beyond eye gaze: What else can eyetracking reveal about cognition and cognitive development? Developmental cognitive neuroscience. 2017;25:69-91.

2. Yarbus AL. Eye Movements During Perception of Complex Objects. Eye Movements and Vision. New York: Plenum Press; 1967.

3. Theeuwes J, Belopolsky A, Olivers $\mathrm{CN}$. Interactions between working memory, attention and eye movements. Acta Psychol (Amst). 2009;132(2):106-14.

4. Thomas LE, Lleras A. Moving eyes and moving thought: on the spatial compatibility between eye movements and cognition. Psychon Bull Rev. 2007;14(4):663-8.

5. Van der Stigchel S, Meeter M, Theeuwes J. Eye movement trajectories and what they tell us. Neurosci Biobehav Rev. 2006;30(5):666-79.

6. Just MA, Carpenter PA. Eye fixations and cognitive processes. 1975.

7. Awh E, Armstrong KM, Moore T. Visual and oculomotor selection: links, causes and implications for spatial attention. Trends Cogn Sci. 2006;10(3):124-30.

8. Grosbras $\mathrm{MH}$, Laird AR, Paus T. Cortical regions involved in eye movements, shifts of attention, and gaze perception. Hum Brain Mapp. 2005;25(1):140-54.

9. Luna B, Velanova K, Geier CF. Development of eye-movement control. Brain Cogn. 2008;68(3):293308.

10. Hoppe D, Rothkopf CA. Learning rational temporal eye movement strategies. Proc Natl Acad Sci U S A. 2016;113(29):8332-7.

11. Vullings $\mathrm{C}$, Madelain L. Control of saccadic latency in a dynamic environment: allocation of saccades in time follows the matching law. J Neurophysiol. 2018;119(2):413-21.

12. Sabatinelli D, Fortune EE, Li Q, Siddiqui A, Krafft C, Oliver WT, et al. Emotional perception: metaanalyses of face and natural scene processing. Neuroimage. 2011;54(3):2524-33.

13. Yucel GH, Belger A, Bizzell J, Parlier M, Adolphs R, Piven J. Abnormal neural activation to faces in the parents of children with autism. Cereb Cortex. 2015;25(12):4653-66.

14. Dalton KM, Nacewicz BM, Johnstone T, Schaefer HS, Gernsbacher MA, Goldsmith HH, et al. Gaze fixation and the neural circuitry of face processing in autism. Nat Neurosci. 2005;8(4):519-26. 
15. Adolphs R. What does the amygdala contribute to social cognition? Ann N Y Acad Sci. 2010;1191:42-61.

16. Kanwisher $\mathrm{N}, \mathrm{McD}$ ermott $\mathrm{J}$, Chun MM. The fusiform face area: a module in human extrastriate cortex specialized for face perception. J Neurosci. 1997;17(11):4302-11.

17. Klin A, Jones W, Schultz R, Volkmar F, Cohen D. Visual fixation patterns during viewing of naturalistic social situations as predictors of social competence in individuals with autism. Arch Gen Psychiatry. 2002;59(9):809-16.

18. Pelphrey KA, Sasson NJ, Reznick JS, Paul G, Goldman BD, Piven J. Visual scanning of faces in autism. J Autism Dev Disord. 2002;32(4):249-61.

19. Klin A, Jones W, Schultz R, Volkmar F, Cohen D. Defining and quantifying the social phenotype in autism. Am J Psychiatry. 2002;159(6):895-908.

20. Chita-Tegmark M. Social attention in ASD: a review and meta-analysis of eye-tracking studies. Res Dev Disabil. 2016;48:79-93.

21. Frazier TW, Strauss M, Klingemier EW, Zetzer EE, Hardan AY, Eng C, et al. A meta-analysis of gaze differences to social and nonsocial information between individuals with and without autism. $\mathrm{J}$ Am Acad Child Adolesc Psychiatry. 2017;56(7):546-55.

22. Papagiannopoulou EA, Chitty KM, Hermens DF, Hickie IB, Lagopoulos J. A systematic review and meta-analysis of eye-tracking studies in children with autism spectrum disorders. Soc Neurosci. 2014;9(6):610-32.

23. Lee M, Nayar K, Maltman N, Hamburger D, Martin GE, Gordon PC, et al. Understanding social communication differences in autism spectrum disorder and first-degree relatives: a study of looking and speaking. J Autism Dev Disord. 2019.

24. Navab A, Gillespie-Lynch K, Johnson SP, Sigman M, Hutman T. Eye-Tracking as a measure of responsiveness to joint attention in infants at risk for autism. Infancy. 2012;17(4):416-31.

25. Righi G, Tenenbaum EJ, McCormick C, Blossom M, Amso D, Sheinkopf SJ. Sensitivity to audiovisual synchrony and its relation to language abilities in children with and without ASD. Autism Res. 2018;11(4):645-53.

26. Speer LL, Cook AE, McMahon WM, Clark E. Face processing in children with autism: effects of stimulus contents and type. Autism. 2007;11(3):265-77.

27. Nayar K, Gordon PC, Martin GE, Hogan AL, La Valle C, McKinney W, et al. Links between looking and speaking in autism and first-degree relatives: insights into the expression of genetic liability to autism. Mol Autism. 2018;9:51. 
28. Sasson NJ, Turner-Brown LM, Holtzclaw TN, Lam KS, Bodfish JW. Children with autism demonstrate circumscribed attention during passive viewing of complex social and nonsocial picture arrays. Autism Res. 2008;1(1):31-42.

29. Chawarska K, Shic F. Looking but not seeing: atypical visual scanning and recognition of faces in 2 and 4-year-old children with autism spectrum disorder. J Autism Dev Disord. 2009;39(12):1663-72.

30. Falck-Ytter T, Fernell E, Gillberg C, von Hofsten C. Face scanning distinguishes social from communication impairments in autism. Dev Sci. 2010;13(6):864-75.

31. Jones W, Carr K, Klin A. Absence of preferential looking to the eyes of approaching adults predicts level of social disability in 2-year-old toddlers with autism spectrum disorder. Arch Gen Psychiatry. 2008;65(8):946-54.

32. Nakano T, Tanaka K, Endo Y, Yamane $Y$, Yamamoto T, Nakano $Y$, et al. Atypical gaze patterns in children and adults with autism spectrum disorders dissociated from developmental changes in gaze behaviour. Proc Biol Sci. 2010;277(1696):2935-43.

33. Chawarska K, Volkmar F, Klin A. Limited attentional bias for faces in toddlers with autism spectrum disorders. Arch Gen Psychiatry. 2010;67(2):178-85.

34. Klin A, Jones W. Altered face scanning and impaired recognition of biological motion in a 15month-old infant with autism. Dev Sci. 2008;11(1):40-6.

35. Klin A, Lin DJ, Gorrindo P, Ramsay G, Jones W. Two-year-olds with autism orient to non-social contingencies rather than biological motion. Nature. 2009;459(7244):257-61.

36. Shic F, Bradshaw J, Klin A, Scassellati B, Chawarska K. Limited activity monitoring in toddlers with autism spectrum disorder. Brain Res. 2011;1380:246-54.

37. Unruh KE, Sasson NJ, Shafer RL, Whitten A, Miller SJ, Turner-Brown L, et al. Social orienting and attention is influenced by the presence of competing nonsocial information in adolescents with autism. Front Neurosci. 2016;10:586.

38. Wang Q, Campbell DJ, Macari SL, Chawarska K, Shic F. Operationalizing atypical gaze in toddlers with autism spectrum disorders: a cohesion-based approach. Mol Autism. 2018;9:25.

39. Chevallier C, Parish-Morris J, McVey A, Rump KM, Sasson NJ, Herrington JD, et al. Measuring social attention and motivation in autism spectrum disorder using eye-tracking: Stimulus type matters. Autism Res. 2015;8(5):620-8.

40. Chawarska K, Macari S, Shic F. Context modulates attention to social scenes in toddlers with autism. J Child Psychol Psychiatry. 2012;53(8):903-13. 
41. Shic F, Chawarska K, Bradshaw J, Scassellati B. Autism, Eye-Tracking, Entropy. 2008 leee 7th International Conference on Development and Learning2008. p. 73-8.

42. Constantino JN, Kennon-McGill S, Weichselbaum C, Marrus N, Haider A, Glowinski AL, et al. Infant viewing of social scenes is under genetic control and is atypical in autism. Nature. 2017;547(7663):340-4.

43. Ettinger U, Kumari V, Crawford TJ, Corr PJ, Das M, Zachariah E, et al. Smooth pursuit and antisaccade eye movements in siblings discordant for schizophrenia. J Psychiatr Res. 2004;38(2):177-84.

44. Gottesman II, Gould TD. The endophenotype concept in psychiatry: etymology and strategic intentions. Am J Psychiatry. 2003;160(4):636-45.

45. Bradshaw J, Shic F, Holden AN, Horowitz EJ, Barrett AC, German TC, et al. The use of eye tracking as a biomarker of treatment outcome in a pilot randomized clinical trial for young children with autism. Autism Res. 2019;12(5):779-93.

46. Murias M, Major S, Davlantis K, Franz L, Harris A, Rardin B, et al. Validation of eye-tracking measures of social attention as a potential biomarker for autism clinical trials. Autism Res. 2018;11(1):166-74.

47. Pierce K, Marinero S, Hazin R, McKenna B, Barnes CC, Malige A. Eye tracking reveals abnormal visual preference for geometric images as an early biomarker of an autism spectrum disorder subtype associated with increased symptom severity. Biol Psychiatry. 2016;79(8):657-66.

48. Adolphs R, Spezio ML, Parlier M, Piven J. Distinct face-processing strategies in parents of autistic children. Curr Biol. 2008;18(14):1090-3.

49. Losh M, Childress D, Lam K, Piven J. Defining key features of the broad autism phenotype: a comparison across parents of multiple- and single-incidence autism families. Am J Med Genet B Neuropsychiatr Genet. 2008;147B(4):424-33.

50. Losh M, Piven J. Social-cognition and the broad autism phenotype: identifying genetically meaningful phenotypes. J Child Psychol Psychiatry. 2007;48(1):105-12.

51. Groen WB, Rommelse N, de Wit T, Zwiers MP, van Meerendonck D, van der Gaag RJ, et al. Visual scanning in very young children with autism and their unaffected parents. Autism Res Treat. 2012;2012:748467.

52. Wass SV, Forssman L, Leppänen J. Robustness and precision: How data quality may influence key dependent variables in infant eye-tracker analyses. Infancy. 2014;19(5):427-60.

53. Wass SV, Smith TJ, Johnson MH. Parsing eye-tracking data of variable quality to provide accurate fixation duration estimates in infants and adults. Behav Res Methods. 2013;45(1):229-50. 
54. Shic F, Chawarska K, Scassellati B. The Incomplete Fixation Measure. 2008.

55. Hughes C, Russell J. Autistic children's difficulty with mental disengagement from an object: Its implications for theories of autism. Developmental psychology. 1993;29(3):498.

56. Booth RW, Weger UW. The function of regressions in reading: backward eye movements allow rereading. Mem Cognit. 2013;41(1):82-97.

57. Perea M, Carreiras M. Regressions and eye movements: where and when. Behavioral and Brain Sciences. 2003;26(4):497-.

58. Rayner K. Eye movements in reading and information processing: 20 years of research. Psychol Bull. 1998;124(3):372-422.

59. Rayner K, Slattery TJ, Belanger NN. Eye movements, the perceptual span, and reading speed. Psychon Bull Rev. 2010;17(6):834-9.

60. Shic F, Chawarska K, Scassellati B. The Amorphous Fixation Measure Revisited: with Applications to Autism. 2008.

61. Jones W, Klin A. Attention to eyes is present but in decline in 2-6-month-old infants later diagnosed with autism. Nature. 2013;504(7480):427-31.

62. Lord C, Rutter M, DiLavore P, Risi S, Gotham K, Bishop S. Autism diagnostic observation schedule2nd edition (ADOS-2). Los Angeles, CA: Western Psychological Corporation. 2012.

63. Lord C, Rutter M, Le Couteur A. Autism Diagnostic Interview-Revised: a revised version of a diagnostic interview for caregivers of individuals with possible pervasive developmental disorders. Journal of autism and developmental disorders. 1994;24(5):659-85.

64. Piven J, Palmer P, Landa R, Santangelo S, Jacobi D, Childress D. Personality and language characteristics in parents from multiple-incidence autism families. Am J Med Genet. 1997;74(4):398-411.

65. Sasson NJ, Lam KS, Childress D, Parlier M, Daniels JL, Piven J. The broad autism phenotype questionnaire: prevalence and diagnostic classification. Autism Res. 2013;6(2):134-43.

66. Murray HA. Thematic apperception test. Cambridge, MA: Harvard University Press; 1943.

67. Hiraishi $\mathrm{H}$, Haida M, Matsumoto M, Hayakawa N, Inomata S, Matsumoto H. Differences of prefrontal cortex activity between picture-based personality tests: a near-infrared spectroscopy study. J Pers Assess. 2012;94(4):366-71.

68. Turk AA, Brown WS, Symington M, Paul LK. Social narratives in agenesis of the corpus callosum: linguistic analysis of the Thematic Apperception Test. Neuropsychologia. 2010;48(1):43-50. 
69. Beaumont R, Newcombe P. Theory of mind and central coherence in adults with high-functioning autism or Asperger syndrome. Autism. 2006;10(4):365-82.

70. Lee M, Martin GE, Hogan A, Hano D, Gordon PC, Losh M. What's the story? A computational analysis of narrative competence in autism. Autism. 2018;22(3):335-44.

71. Anderson CJ, Colombo J, Jill Shaddy D. Visual scanning and pupillary responses in young children with Autism Spectrum Disorder. J Clin Exp Neuropsychol. 2006;28(7):1238-56.

72. Dink JW, Ferguson B. eyetrackingR: An R Library for Eye-tracking Data Analysis. 2015 [Available from: http://www.eyetrackingr.com.

73. Demarais AM, Cohen BH. Evidence for image-scanning eye movements during transitive inference. Biol Psychol. 1998;49(3):229-47.

74. Thibaut JP, French RM. Analogical reasoning, control and executive functions: a developmental investigation with eye-tracking. Cognitive Development. 2016;38:10-26.

75. Vigneau F, Caissie AF, Bors DA. Eye-movement analysis demonstrates strategic influences on intelligence. Intelligence. 2006;34(3):261-72.

76. Kimchi R. Primacy of wholistic processing and global/local paradigm: a critical review. Psychol Bull. 1992;112(1):24-38.

77. Zagermann J, Pfeil U, Reiterer $\mathrm{H}$, editors. Studying eye movements as a basis for measuring cognitive load. Extended Abstracts of the $2018 \mathrm{CHI}$ Conference on Human Factors in Computing Systems; 2018.

78. Hessels RS, Kemner C, van den Boomen C, Hooge IT. The area-of-interest problem in eyetracking research: A noise-robust solution for face and sparse stimuli. Behav Res Methods. 2016;48(4):1694-712.

79. Olson CL. Comparative robustness of six tests in multivariate analysis of variance. Journal of the American Statistical Association. 1974;69(348):894-908.

80. Winston M, Nayar K, Hogan AL, Barstein J, La Valle C, Sharp K, et al. Physiological regulation and social-emotional processing in female carriers of the FMR1 premutation. Physiology \& behavior. 2020;214:112746.

81. Lever J, Krzywinski M., Altman N. Principal component analysis. Nature Methods. 2017;14:641-2.

82. Van der Hallen R, Evers K, Brewaeys K, Van den Noortgate W, Wagemans J. Global processing takes time: a meta-analysis on local-global visual processing in ASD. Psychol Bull. 2015;141(3):549-73.

83. McPartland JC, Webb SJ, Keehn B, Dawson G. Patterns of visual attention to faces and objects in autism spectrum disorder. J Autism Dev Disord. 2011;41(2):148-57. 
84. McPartland J, Dawson G, Webb SJ, Panagiotides H, Carver LJ. Event-related brain potentials reveal anomalies in temporal processing of faces in autism spectrum disorder. J Child Psychol Psychiatry. 2004;45(7):1235-45.

85. O'Connor K, Kirk I. Brief Report: Atypical Social Cognition and Social Behaviours in Autism Spectrum Disorder: A Different Way of Processing Rather than an Impairment. Journal of Autism and Developmental Disorders. 2008;38(10):1989-97.

86. Swanson MR, Serlin GC, Siller M. Broad autism phenotype in typically developing children predicts performance on an eye-tracking measure of joint attention. J Autism Dev Disord. 2013;43(3):707-18.

87. Au-Yeung SK, Benson V, Castelhano M, Rayner K. Eye movement sequences during simple versus complex information processing of scenes in autism spectrum disorder. Autism Res Treat. 2011;2011:657383.

88. Nayar K, Kang X, Xing J, Gordon PC, Wong PCM, Losh M. A cross-cultural study showing deficits in gaze-language coordination during rapid automatized naming among individuals with ASD. Sci Rep. 2021;11(1):13401.

89. Cuccaro ML, Shao Y, Grubber J, Slifer M, Wolpert CM, Donnelly SL, et al. Factor analysis of restricted and repetitive behaviors in autism using the Autism Diagnostic Interview-R. Child psychiatry and human development. 2003;34(1):3-17.

90. Lam KSL, Bodfish JW, Piven J. Evidence for three subtypes of repetitive behavior in autism that differ in familiality and association with other symptoms. Journal of Child Psychology and Psychiatry. 2008;49(11):1193-200.

91. Richler J, Bishop SL, Kleinke JR, Lord C. Restricted and repetitive behaviors in young children with autism spectrum disorders. J Autism Dev Disord. 2007;37(1):73-85.

92. Losh M, Adolphs R, Piven J. The broad autism phenotype. Autism spectrum disorders: Oxford University Press; 2011.

93. Losh M, Klusek J, Martin GE, Sideris J, Parlier M, Piven J. Defining genetically meaningful language and personality traits in relatives of individuals with fragile $X$ syndrome and relatives of individuals with autism. Am J Med Genet B Neuropsychiatr Genet. 2012;159B(6):660-8.

94. Losh M, Adolphs R, Poe MD, Couture S, Penn D, Baranek GT, et al. Neuropsychological profile of autism and the broad autism phenotype. Arch Gen Psychiatry. 2009;66(5):518-26.

95. Piven J, Wzorek M, Landa R, Lainhart J, Bolton P, Chase GA, et al. Personality characteristics of the parents of autistic individuals. Psychol Med. 1994;24(3):783-95. 
96. Van Eylen L, Boets B, Steyaert J, Wagemans J, Noens I. Local and Global Visual Processing in Autism Spectrum Disorders: Influence of Task and Sample Characteristics and Relation to Symptom Severity. J Autism Dev Disord. 2018;48(4):1359-81.

97. Plaisted K, Swettenham J, Rees L. Children with autism show local precedence in a divided attention task and global precedence in a selective attention task. J Child Psychol Psychiatry. 1999;40(5):733-42.

98. Rinehart NJ, Bradshaw JL, Moss SA, Brereton AV, Tonge BJ. Atypical interference of local detail on global processing in high-functioning autism and Asperger's disorder. J Child Psychol Psychiatry. 2000;41(6):769-78.

99. Grandin T. Thinking in pictures and other reports from my life with autism1995.

100. Franchini M, Glaser B, de Wilde HW, Gentaz E, Eliez S, Schaer M. Social orienting and joint attention in preschoolers with autism spectrum disorders. Plos One. 2017;12(6).

101. Harrop C, Jones D, Zheng S, Nowell S, Boyd BA, Sasson N. Circumscribed interests and attention in autism: the role of biological sex. J Autism Dev Disord. 2018;48(10):3449-59.

102. Fischer J, Koldewyn K, Jiang YV, Kanwisher N. Unimpaired Attentional Disengagement and Social Orienting in Children with Autism. Clin Psychol Sci. 2014;2(2):214-23.

103. Holmqvist K, Nyström M, Andersson R, Dewhurst R, Jarodzka H, Weijer JVD. A comprehensive guide to methods and measure. Oxford, U: Oxford University Press; 2011.

\section{Figures}


A

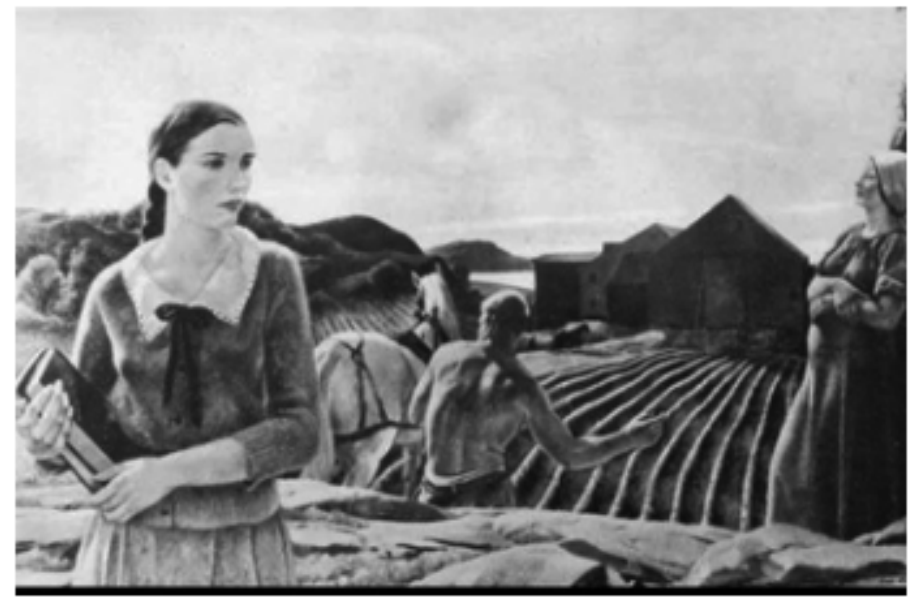

B

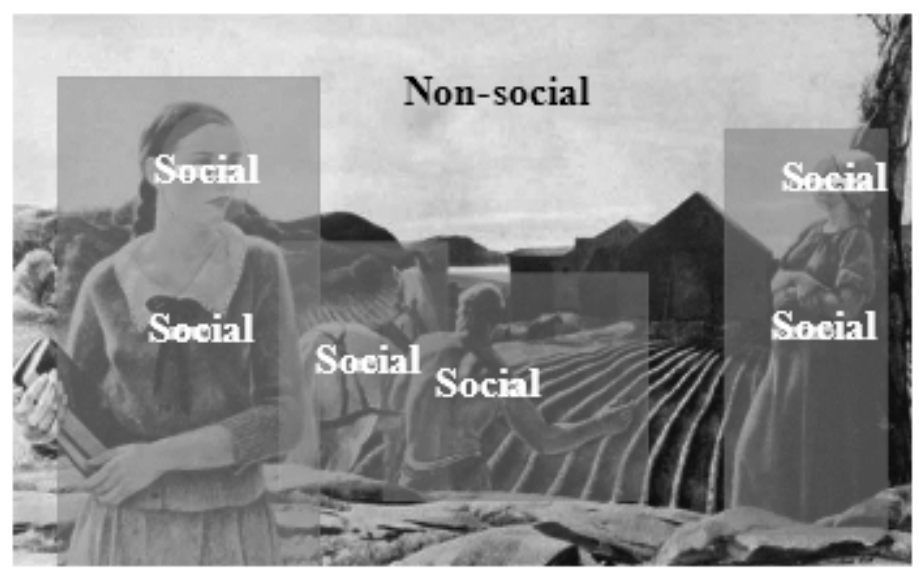

Figure 1

A) TAT image examined-Card 2; Farmland Scene; B) Two primary areas of interest (AOls) were generated -Social AOI, which included all the characters in the image; and Non-social AOI, which included everything else such as the book, barn, field. 
A

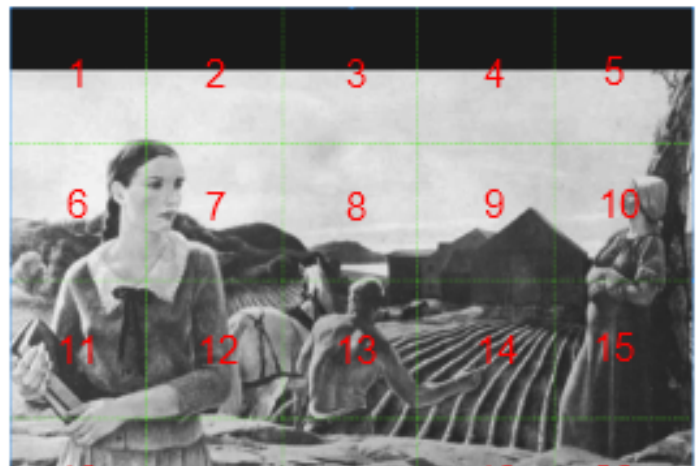

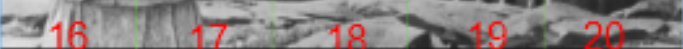

B

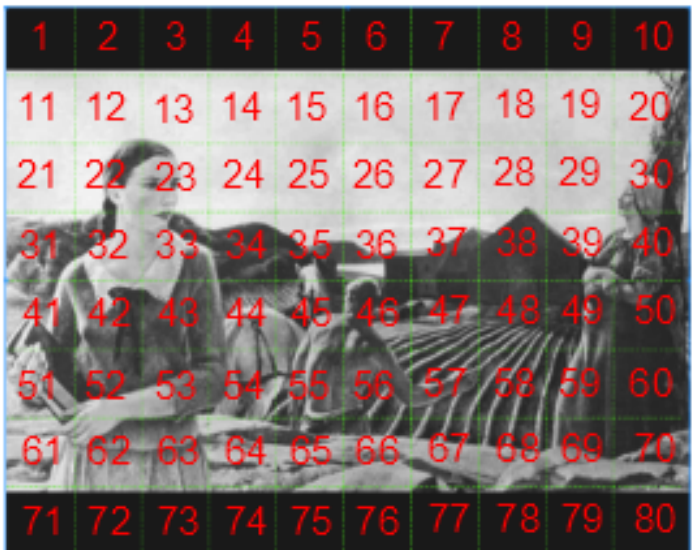

\section{Figure 2}

Schematic representing fixation distribution scatter/coverage analysis AOls. A) large areas (5 x 4 grid) and $B)$ small areas (10 $\times 8$ grid). 
A
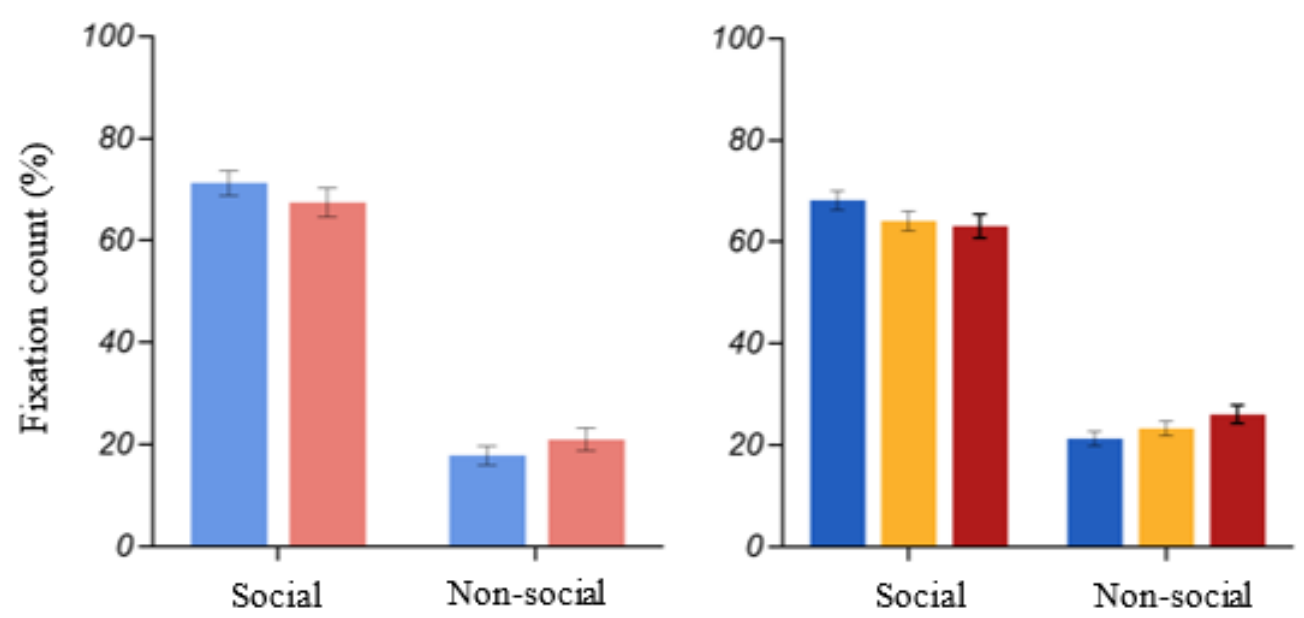

B
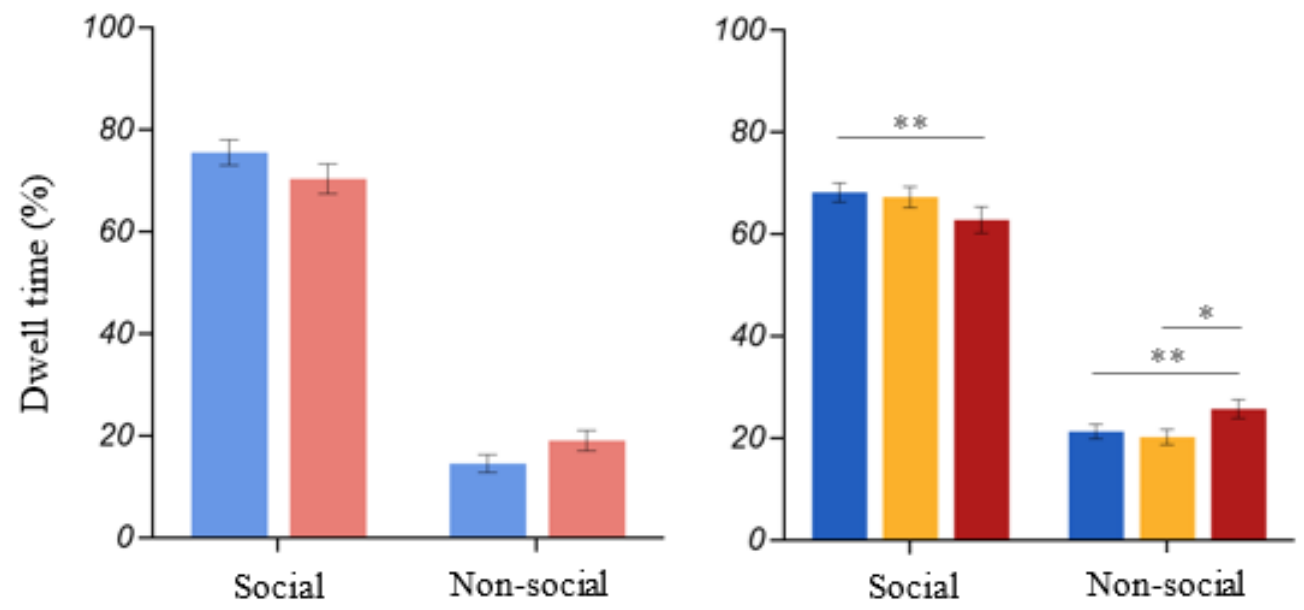

${ }^{*} p<.05$

$* * p<.01$

- Control group

- ASD group

- Control parent group

- $\mathrm{BAP}(-)$ parent group

- $\mathrm{BAP}(+)$ parent group

\section{Figure 3}

Overall gaze variables depicting A) fixation count and B) dwell time. Significant differences between $\mathrm{BAP}(+)$ and Control parent groups emerged in dwell time across social and non-social visual attention. 
A
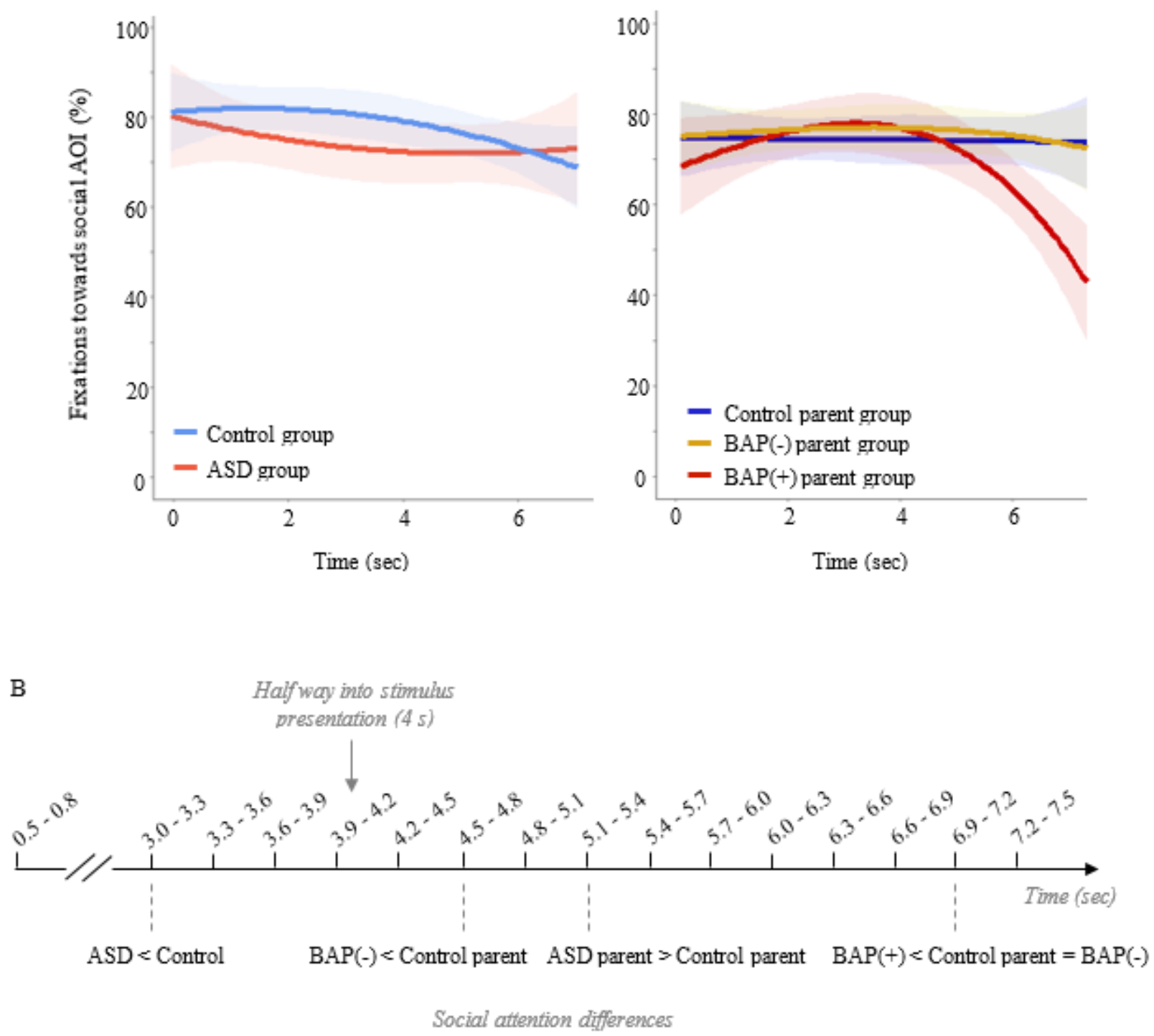

Figure 4

Dwell time patterns depicting A) proportion of fixations over time (higher value indicates greater social attention) and B) a schematic representing divergence time-bin analyses, where individuals with ASD were observed to attend less to social information than the control group half way into the stimulus presentation. Both $\mathrm{BAP}(-)$ and $\mathrm{BAP}(+)$ parents showed a spike in social attention around 5 seconds, with the $\mathrm{BAP}(+)$ group showing a striking decrease in social attention towards the end of the stimulus presentation compared to BAP(-) and Control parent groups. 
A
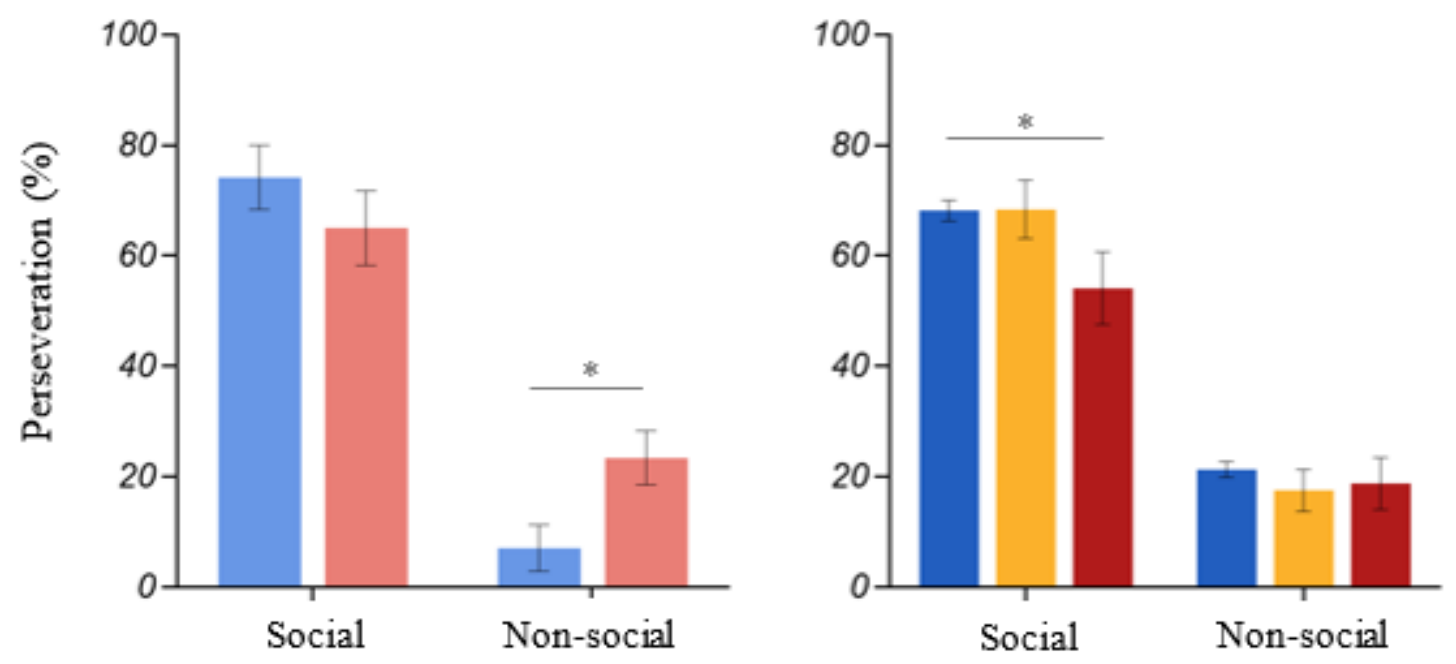

B
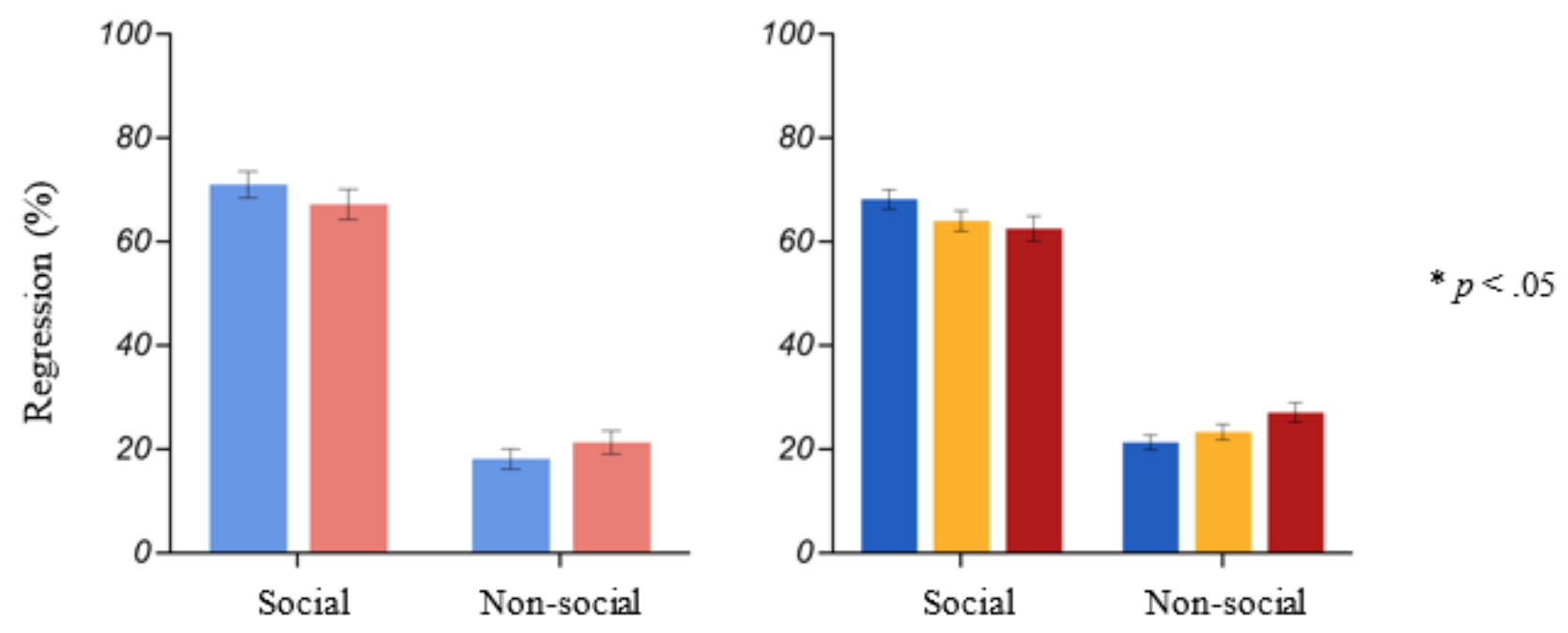

Figure 5

Fixation patterns depicting A) Percentage of perseverative fixations and B) Percentage of regressive fixations. Significant differences between ASD and control groups, and BAP(+) and Control parent groups emerged in perseverative fixation patterns, showing elevated non-social and reduced social perseverative fixations, respectively. 

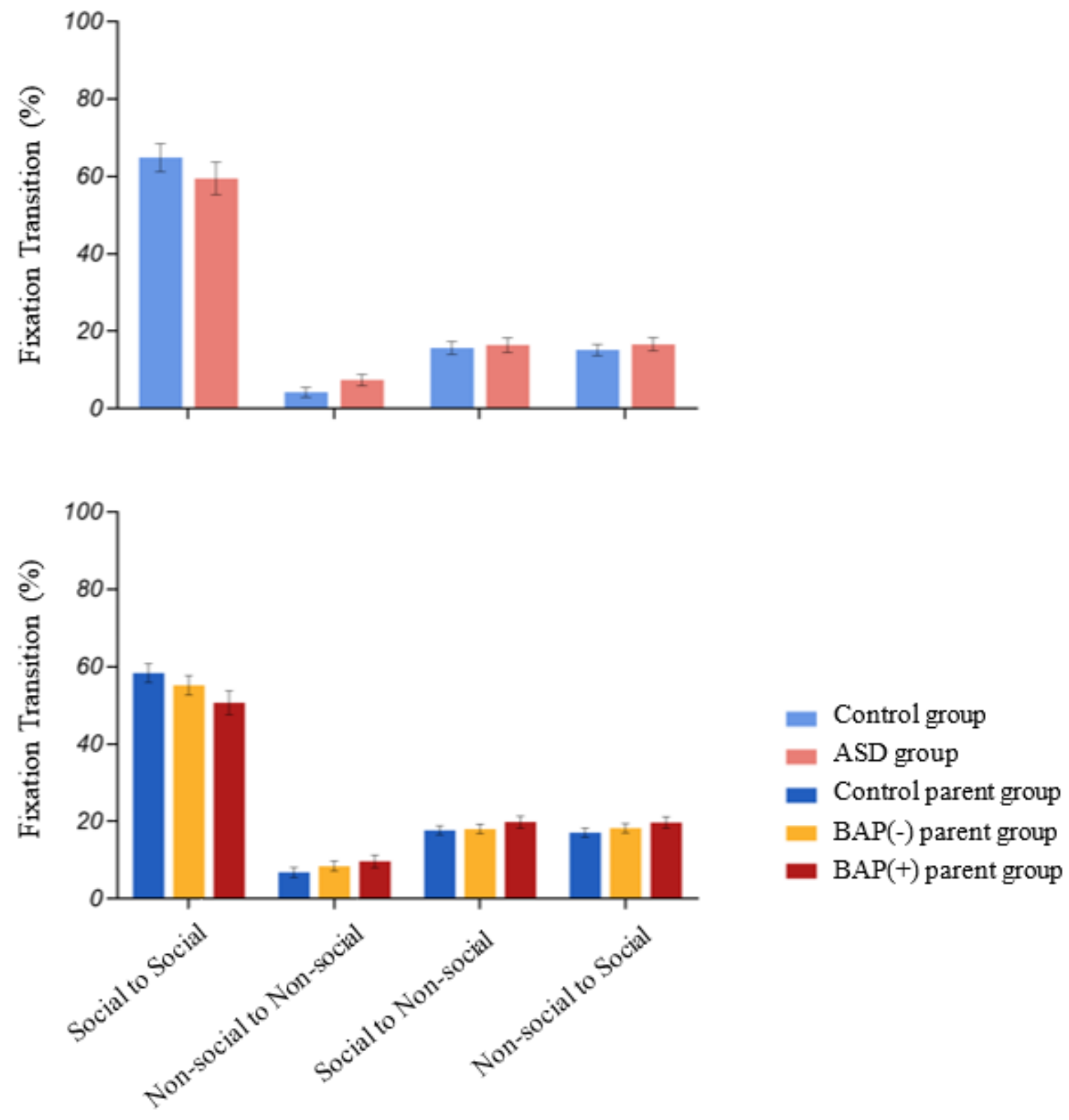

Figure 6

Fixation patterns depicting transition analysis (i.e., the percentage of fixations characterized as transitions from one AOI to another) as follows: social to social AOI, non-social to non-social AOI, social to non-social AOI, and non-social to social AOI transitions. 
A

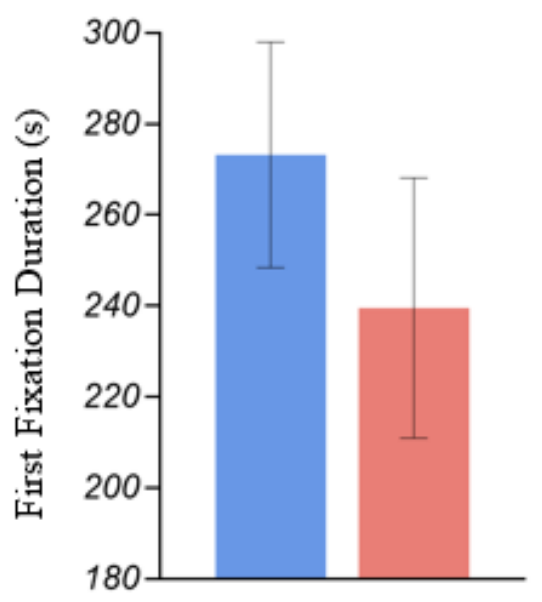

B

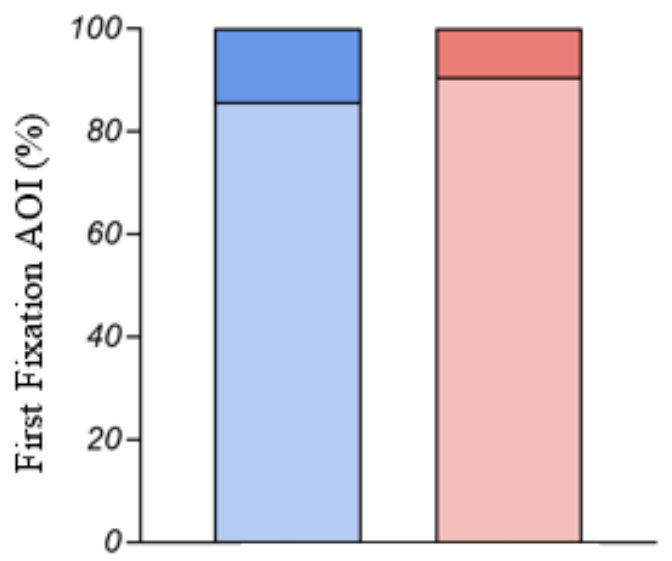

300
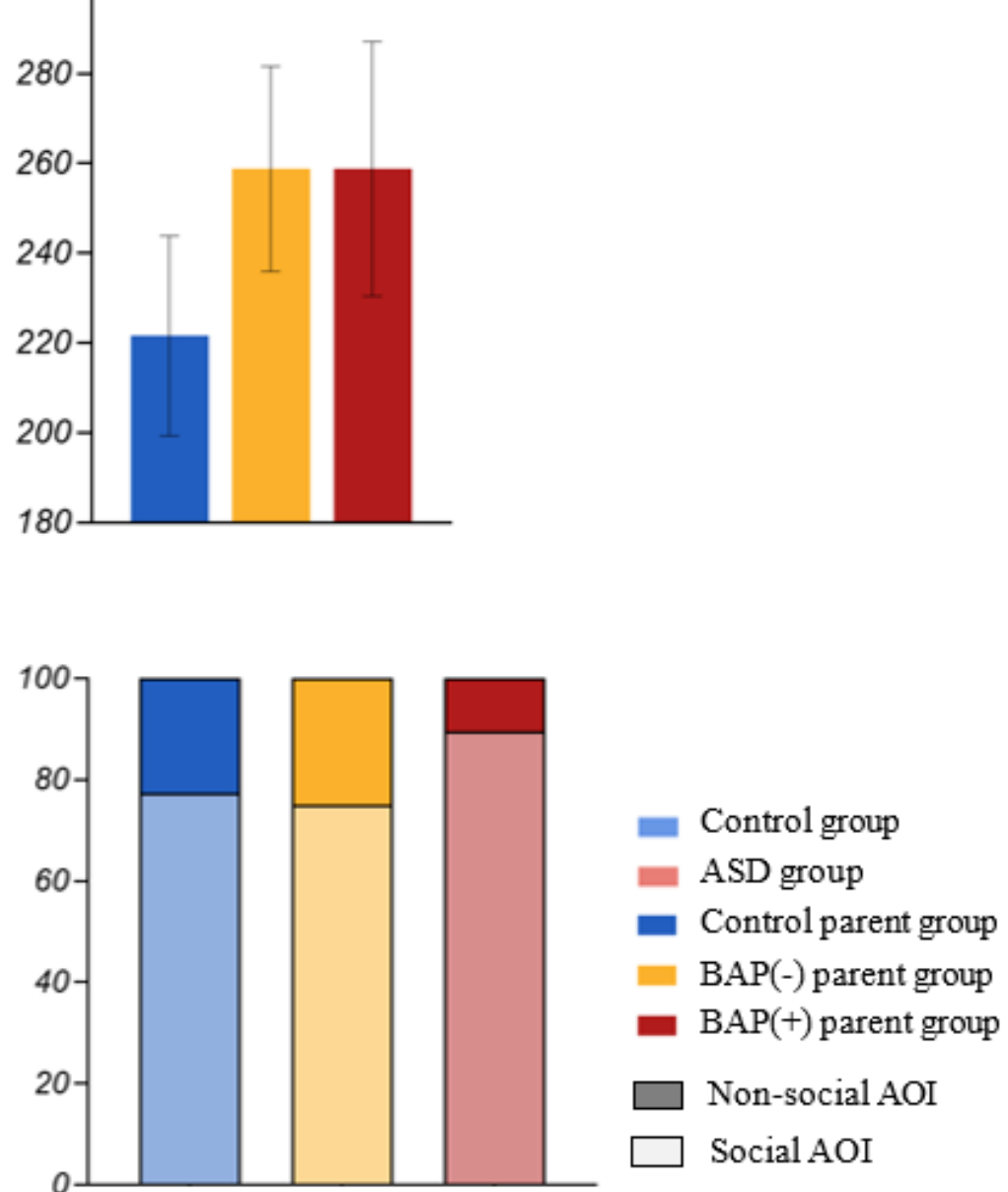

Figure 7

A) First fixation duration, and B) First fixation AOI showing both percentage of first fixations that were made towards social information (lighter shade) and non-social information (darker shade). 
A
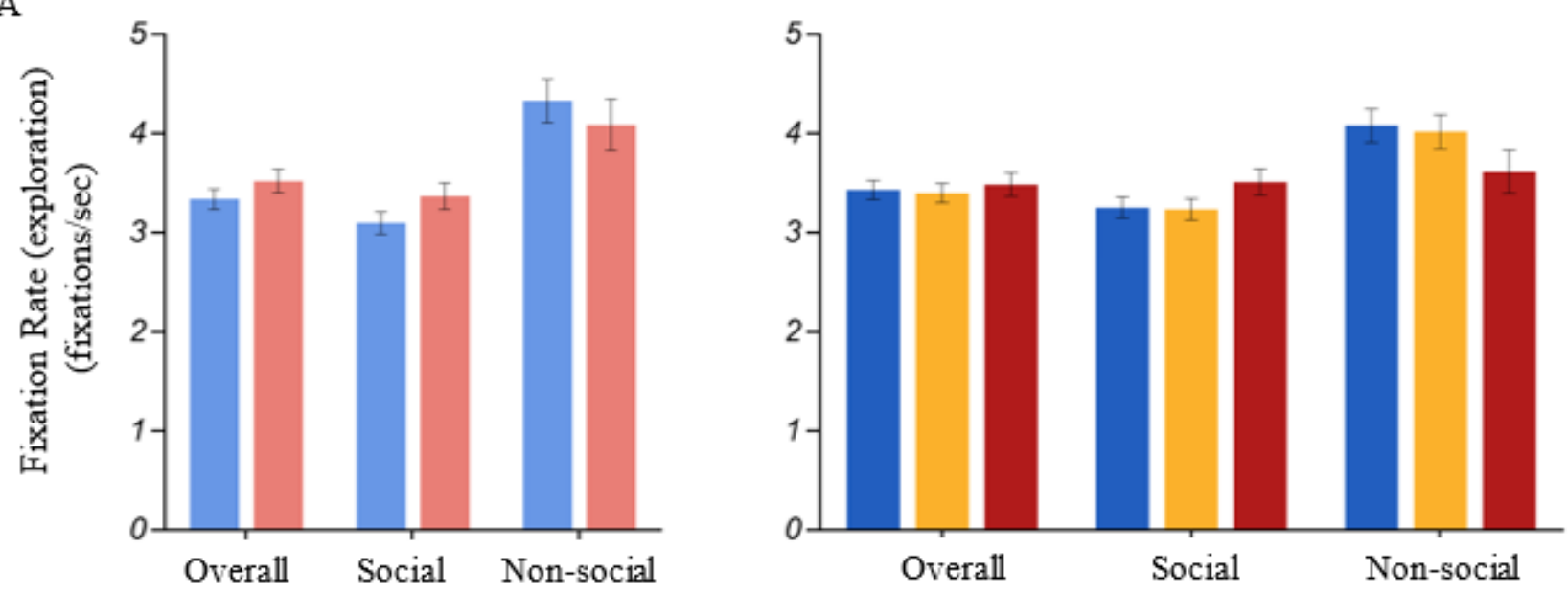

B
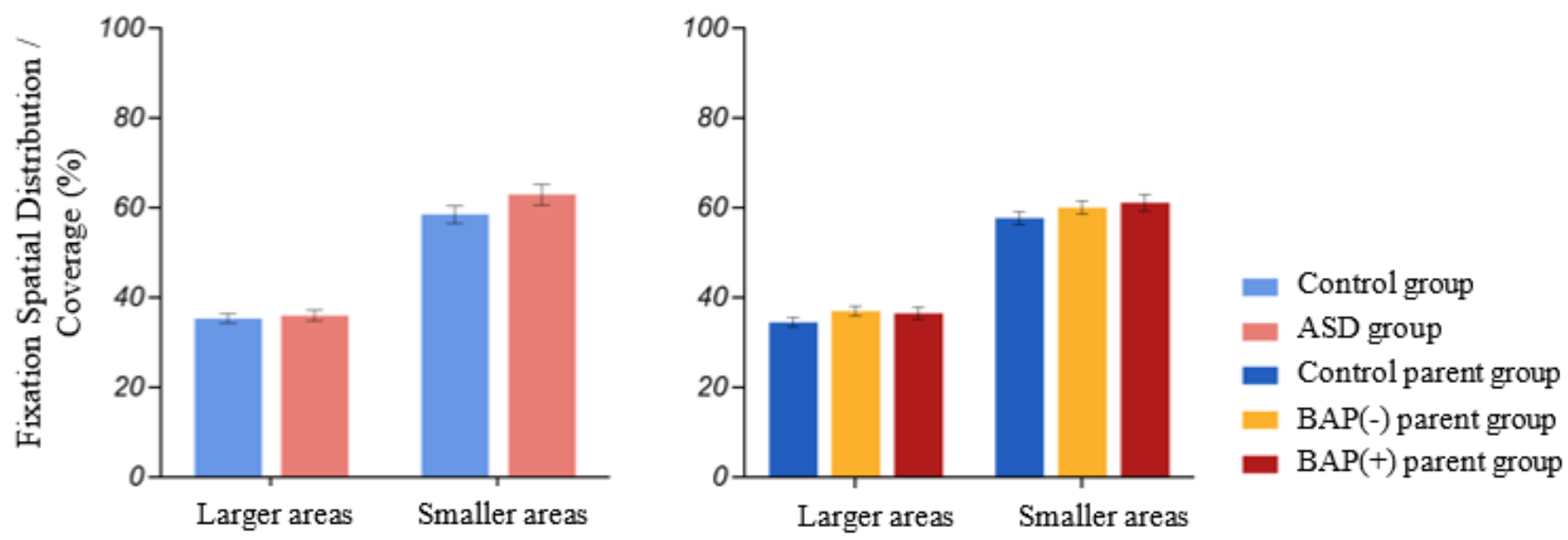

Figure 8

Distribution analyses depicting both A) Fixation rate (exploration) overall and by AOI (social and nonsocial), and B) fixation spatial distribution/coverage across larger (5 x 4) and smaller (10 x 8) boxes. 


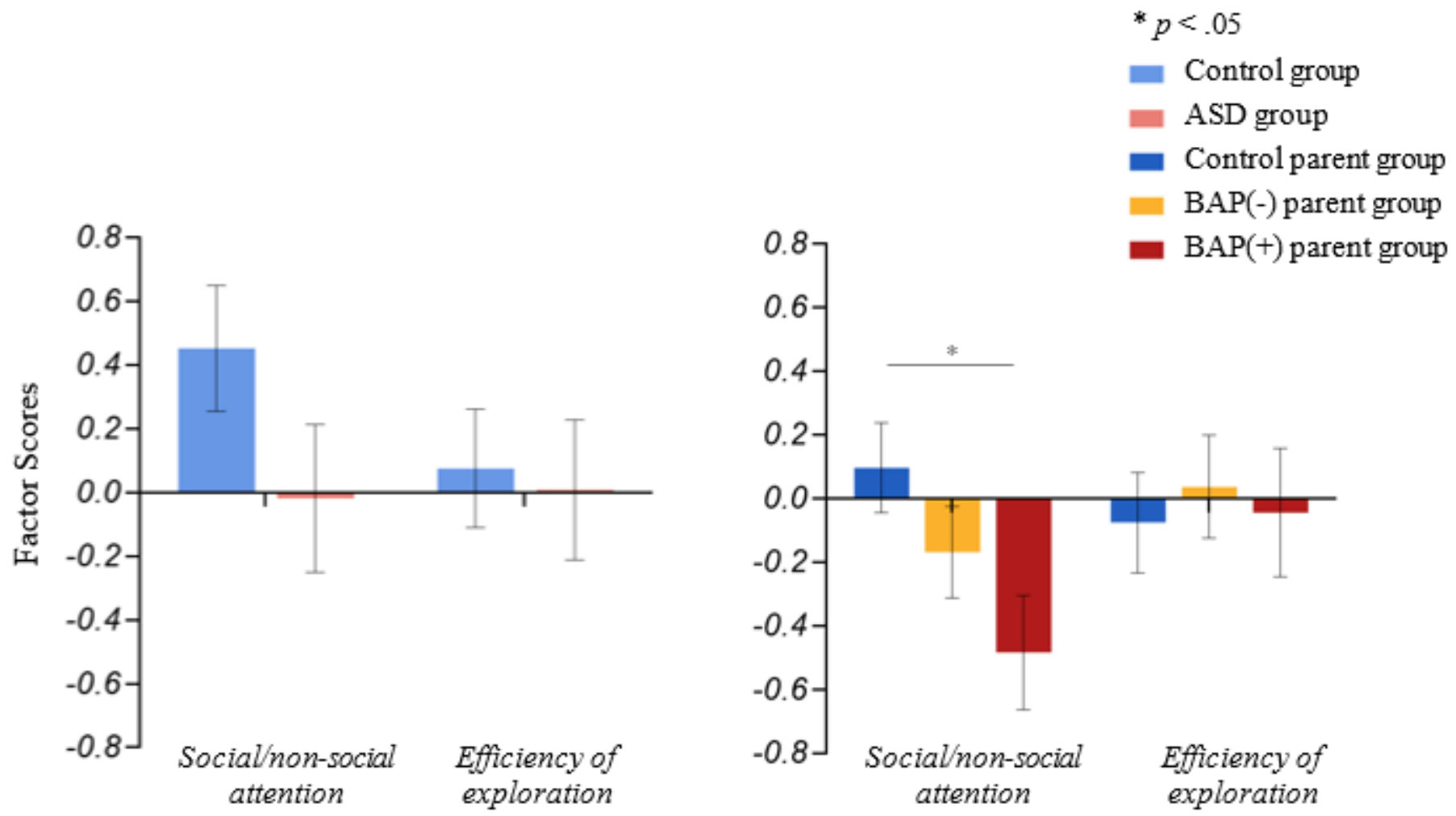

\section{Figure 9}

Group differences in factor scores from principal component analysis. Factor $1=$ social $/$ non-social attention (higher scores indicate greater social looking); factor 2 = efficiency of exploration.

\section{Supplementary Files}

This is a list of supplementary files associated with this preprint. Click to download.

- SupplementaryMaterials.docx 